\title{
Physical conditions of molecular gas in the Circinus galaxy Multi-J $\mathrm{CO}$ and $\mathrm{CI}^{3} \mathrm{P}_{\mathbf{1}} \rightarrow{ }^{3} \mathrm{P}_{\mathbf{0}}$ observations ${ }^{\star}$
}

\author{
Zhi-Yu Zhang ${ }^{1,2,3,8}$, Christian Henkel ${ }^{2,4}$, Yu Gao $^{1}$, Rolf Güsten ${ }^{2}$, Karl M. Menten ${ }^{2}$, Padelis P. Papadopoulos ${ }^{5}$, \\ Yinghe Zhao ${ }^{6,1}$, Yiping Ao ${ }^{7,2,1}$, and Tomasz Kaminski ${ }^{2}$

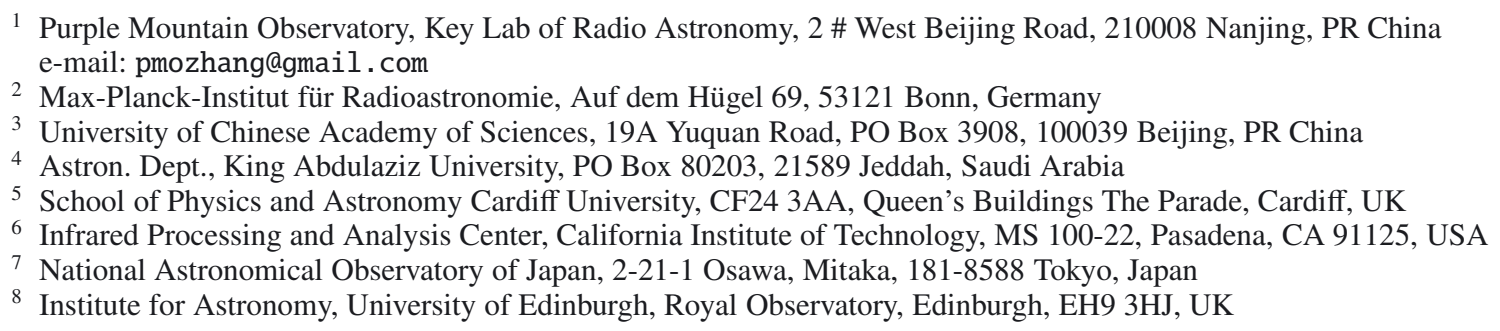

Received 10 September 2013 / Accepted 4 June 2014

\section{ABSTRACT}

\begin{abstract}
We report mapping observations of the ${ }^{12} \mathrm{CO} J=3 \rightarrow 2,4 \rightarrow 3,6 \rightarrow 5$, and $7 \rightarrow 6$ transitions and the C I ${ }^{3} \mathrm{P}_{1} \rightarrow{ }^{3} \mathrm{P}_{0}(\mathrm{CI}) 492 \mathrm{GHz}$ transition toward the central $40^{\prime \prime} \times 40^{\prime \prime}$ region of the Circinus galaxy, using the Atacama Pathfinder EXperiment (APEX) telescope. We also detected ${ }^{13} \mathrm{CO} J=3 \rightarrow 2$ at the central position of Circinus. These observations are to date the highest $\mathrm{CO}$ transitions reported in Circinus. With large velocity gradient (LVG) modeling and likelihood analysis we try to obtain density, temperature, and column density of the molecular gas in three regions: the nuclear region $\left(D<18^{\prime \prime} \sim 360 \mathrm{pc}\right)$, the entire central $45^{\prime \prime}\left(D<45^{\prime \prime} \sim 900 \mathrm{pc}\right)$ region, and the star-forming (S-F) ring $\left(18^{\prime \prime}<D<45^{\prime \prime}\right)$. In the nuclear region, we can fit the $\mathrm{CO}$ excitation with a single excitation component, yielding an average condition of $n_{\mathrm{H}_{2}} \sim 10^{3.2} \mathrm{~cm}^{-3}, T_{\text {kin }} \sim 200 \mathrm{~K}$, and $\mathrm{d} v / \mathrm{d} r \sim 3 \mathrm{~km} \mathrm{~s}^{-1} \mathrm{pc}^{-1}$. In the entire $45^{\prime \prime}$ region, which covers both the nucleus and the S-F ring, two excitation components are needed with $n_{\mathrm{H}_{2}} \sim 10^{4.2} \mathrm{~cm}^{-3}$ and $10^{3.0} \mathrm{~cm}^{-3}, T_{\text {kin }} \sim$ $60 \mathrm{~K}$ and $30 \mathrm{~K}$, and $M_{\mathrm{H}_{2}} \sim 2.3 \times 10^{7} M_{\odot}$ and $6.6 \times 10^{7} M_{\odot}$, respectively. The gas excitation in the S-F ring can also be fitted with two LVG components, after subtracting the CO fluxes in the $18^{\prime \prime}$ nuclear region. The S-F ring region contributes $80 \%$ of the molecular mass in the $45^{\prime \prime}$ region. For the entire $45^{\prime \prime}$ region, we find a standard conversion factor of $N\left(\mathrm{H}_{2}\right) / I_{\mathrm{CO}} \rightarrow 0=0.37 \times 10^{20} \mathrm{~cm}^{-2}\left(\mathrm{~K} \mathrm{~km} \mathrm{~s}^{-1}\right)^{-1}$, about $1 / 5$ of the Galactic disk value. The luminosity ratios of $\mathrm{CI}$ and ${ }^{12} \mathrm{CO} J=3 \rightarrow 2\left(R_{\mathrm{CI} / \mathrm{CO} 3 \rightarrow 2}\right)$ in Circinus basically follow a linear trend, similar to that obtained in high-redshift galaxies. The average $R_{\mathrm{CI} / \mathrm{CO} \mathrm{J}=3 \rightarrow 2}$ in Circinus is found to be $\sim 0.2$, lying at an intermediate value between non-AGN nuclear regions and high-redshift galaxies.
\end{abstract}

Key words. galaxies: abundances - galaxies: ISM - galaxies: individual: Circinus - galaxies: evolution - radio lines: galaxies

\section{Introduction}

Multiple rotational transitions of $\mathrm{CO}$ are a powerful tool to study the physical environment and the excitation conditions of molecular gas in galaxies. For galaxies harboring active galactic nuclei (AGN), the nuclear activity is often powered by the molecular gas surrounding the nuclear region, and the feedback - jets, winds, and radiation - may enhance or quench the star-forming (S-F) activity (e.g., Bundy et al. 2008; Sani et al. 2010). The excitation of molecular gas in the torus ( $\sim$ a few pc to tens of pc) and in the circumnuclear disk (CND; a few tens to hundreds of pc) reflects the activity invoked by the illumination from the central supermassive black hole (SMBH; e.g., Schinnerer et al. 2000; Pérez-Beaupuits et al. 2011; Harada et al. 2013). Because of its symmetry, molecular hydrogen $\left(\mathrm{H}_{2}\right)$ has no permanent dipole moment and its infrared transitions require high excitation conditions, thus the $\mathrm{H}_{2}$ emission is not able to trace molecular clouds (e.g., Kennicutt \& Evans 2012). Carbon monoxide (CO), the second most abundant molecule, has a dipole moment

\footnotetext{
^ Appendices are available in electronic form at http://www . aanda.org
}

of 1.122 Debye and is heavy enough for a rotational spectrum accessible at submillimeter (submm) wavelengths, tracing both cold and warm gas. CO lines are therefore regarded as the best tracers to the probe the physical properties and the excitation conditions of the entire molecular gas reservoir (e.g., Mao et al. 2000; van der Werf et al. 2010; Papadopoulos et al. 2012b).

So far, in nearby galaxies most studies of the molecular gas emission focus on the $J=1 \rightarrow 0,2 \rightarrow 1$, and $3 \rightarrow 2$ transitions of CO (e.g., Braine et al. 1993; Dumke et al. 2001; Israel \& Baas 2003; Wilson et al. 2011). At high redshifts mid- $J(4 \leq J \leq 8)$ $\mathrm{CO}$ transitions are almost exclusively measured (e.g., Omont et al. 1996; Carilli et al. 2010; Wang et al. 2010). Therefore, observations of the mid- $J$ transitions in some nearby galaxies are essential to investigate the gas excitation, as reference of the high-redshift galaxies. Such studies have been focused on nearby S-F galaxies such as NGC 253, IC 342, and NGC 4038, (e.g., Güsten et al. 2006; Hailey-Dunsheath et al. 2008; Bayet et al. 2009), but only a few nearby galaxies with prominent AGNs have been studied so far in these mid- $J$ CO lines (e.g., NGC 1068; Israel 2009a; Spinoglio et al. 2012).

The Circinus galaxy is a prototypical Seyfert-2 galaxy located in the southern sky, at a small distance of $D \sim 4 \mathrm{Mpc}$ (e.g., 
Maiolino et al. 1998; Tully et al. 2009). Although it has a large angular size $\left(\geq 80^{\prime}\right)$ at optical wavelengths and in atomic gas (HI), Circinus was not discovered until the 1970s (Freeman et al. 1977; Jones et al. 1999), because it is located only $\sim 4^{\circ}$ above the Galactic plane with a Galactic visual extinction of 4.8 mag (e.g., Schlegel et al. 1998). $\mathrm{H}_{2} \mathrm{O}$ mega-masers at both $\mathrm{mm}$ and submm wavelengths were found in the center of Circinus (e.g., Greenhill et al. 2003; Hagiwara et al. 2013), indicating a molecular torus around the central SMBH. The mass of the SMBH is estimated to be $1.7 \pm 0.3 \times 10^{6} M_{\odot}$ (e.g., Greenhill et al. 2003), and the torus accretion rate is as high as $\sim 20 \%$ of the Eddington luminosity (e.g., Tristram et al. 2007).

A large amount of molecular gas in Circinus was detected through the ${ }^{12} \mathrm{COJ}=1 \rightarrow 0$ and ${ }^{13} \mathrm{COJ}=1 \rightarrow 0$ observations by Aalto et al. (1991), with the Swedish-ESO $15 \mathrm{~m}$ Submillimeter Telescope (SEST). Besides the isotopologues of $\mathrm{CO}$ lines (i.e., ${ }^{13} \mathrm{CO}, \mathrm{C}^{18} \mathrm{O}$ ), Curran et al. (2001) contained rich molecular spectra, including lines from $\mathrm{HCN}, \mathrm{HNC}$, and $\mathrm{HCO}^{+}$, which indicate the presence of highly excited dense gas in the central region of Circinus. Furthermore, Circinus was also mapped in the $J=1 \rightarrow 0,2 \rightarrow 1$, and $3 \rightarrow 2$ transitions of CO (Curran et al. 2001, 2008). With the deconvolved ${ }^{12} \mathrm{CO} J=2 \rightarrow 1$ map, Johansson et al. (1991) found a faceon molecular ring structure, which seems to be associated with the S-F ring shown in $\mathrm{H} \alpha$ (Marconi et al. 1994). Curran et al. $(1998,1999)$ found that the gas kinematics could be modeled with a highly inclined molecular ring and two outflows using the ${ }^{12} \mathrm{COJ}=2 \rightarrow 1$ data.

Hitschfeld et al. (2008) observed the ${ }^{12} \mathrm{CO} J=4 \rightarrow 3$ and $\mathrm{C} \mathrm{I}^{3} \mathrm{P}_{1} \rightarrow{ }^{3} \mathrm{P}_{0}$ (hereafter $\mathrm{C} \mathrm{I} 1 \rightarrow 0$ ) lines in the center of Circinus with the NANTEN-2 $4 \mathrm{~m}$ telescope. They studied the molecular gas excitation and predicted that the global $\mathrm{CO}$ cooling curve peaks at the $J=4 \rightarrow 3$ transition, however, higher- $J$ CO transitions are still needed to test their model and to compare the results with other nearby galaxies at similar scales. The turnover of the global CO line ladders is also very important for comparisons with molecular line surveys of gas-rich S-F objects (e.g., Blain et al. 2000; Combes et al. 1999; Geach \& Papadopoulos 2012; Carilli \& Walter 2013). With the large beam sizes of the single dish telescopes in the published low- $J$ observations, it is in any case difficult to explore the excitation conditions in the very central region of Circinus. For these reasons, we have performed high-resolution mapping observations of mid- $J \mathrm{CO}$ lines and the C I $1 \rightarrow 0$ transition in the central region of Circinus.

This article is organized as follows. Section 2 describes the observational methods and data reduction procedure. Section 3 presents the spectra and maps. In Sect.4, the CO lines are analyzed using large velocity gradient (LVG) modeling. The discussion of the data and our modeling results are also presented. In Sect. 5, our findings and conclusions are summarized. Throughout this paper, we adopt for the distance of Circinus a value of 4.2 Mpc (Freeman et al. 1977). Thus 1" corresponds to $\sim 20 \mathrm{pc}$.

\section{Observations and data reduction}

\section{1. $C O$ and $C$ I $1 \rightarrow 0$ spectral line observations}

The observations were performed with the 12-m Atacama Pathfinder EXperiment (APEX) telescope ${ }^{1}$ on the Chajnantor

\footnotetext{
1 This publication is based on data acquired with the Atacama Pathfinder Experiment (APEX). APEX is a collaboration between the Max-Planck-Institut für Radioastronomie, the European Southern Observatory, and the Onsala Space Observatory.
}

Plateau in Chile. Most observations were obtained in good $(\tau<$ 0.3 at $810 \mathrm{GHz}$ ) to median ( $\tau \sim 0.6-1$ at $345 \mathrm{GHz}$ and $460 \mathrm{GHz}$ ) weather conditions during several runs between 2006 and 2010. The C I $1 \rightarrow 0$ data and a part of the ${ }^{12} \mathrm{CO} J=3 \rightarrow 2$ data were taken simultaneously on 14 July, 2010, with the FLASH dual-frequency receiver. ${ }^{12} \mathrm{CO} J=6 \rightarrow 5$ and $7 \rightarrow 6$ maps were obtained simultaneously with the $\mathrm{CHAMP}^{+}$7-pixel receiver array (Kasemann et al. 2006), during June and August 2009. Single point ${ }^{13} \mathrm{CO} J=3 \rightarrow 2$ measurements were taken toward the central position of Circinus $\left(\alpha(J 2000)=14^{\mathrm{h}} 13^{\mathrm{m}} 10.0^{\mathrm{s}}\right.$, $\delta(J 2000)=-65^{\circ} 20^{\prime} 21^{\prime \prime}$. 0$)$ in July 2006 and October 2009. Fast Fourier Transform Spectrometer (FFTS) backends (Klein et al. 2006) were employed in all observations, with channel spacings of 1 or $2 \mathrm{MHz}$, which were then smoothed to suitable velocity resolutions in the data reduction.

We determined focus every four to five hours on Saturn and Jupiter. We calibrated pointing every one to two hours. This ensures $2^{\prime \prime}$ (rms) pointing uncertainties derived from G327.30.6 for ${ }^{12} \mathrm{CO} J=6 \rightarrow 5$ and ${ }^{12} \mathrm{CO} J=7 \rightarrow 6$. The angular resolution varies from $18^{\prime \prime}$ (for ${ }^{12} \mathrm{CO} J=3 \rightarrow 2$ ) to $8^{\prime \prime}$ (for ${ }^{12} \mathrm{CO} J=7 \rightarrow 6$ ), according to the observing frequencies. At the $345 \mathrm{GHz}$ and $460 \mathrm{GHz}$ bands, because there was no suitable strong nearby pointing calibrator at similar elevations, we made pointing calibrations with planets, NGC 6334I (about $40^{\circ}$ away from Circinus), and the strong ${ }^{12} \mathrm{CO} J=3 \rightarrow 2$ emission from Circinus itself. Here we estimate systematic position errors to be $\sim 5^{\prime \prime}$ (rms).

We carried out the mapping observations in raster scan or onthe-fly (OTF) mode. We used a position of $10^{\prime}$ to the east of the center of Circinus as the sky reference. Except for ${ }^{13} \mathrm{CO} J=3 \rightarrow$ 2 , which was only measured at the central position of Circinus, all CO lines are fully sampled in the innermost $\sim 40^{\prime \prime} \times 40^{\prime \prime}$ $(\sim 800 \times 800 \mathrm{pc})$ with Nyquist sampling. The mapped sizes of ${ }^{12} \mathrm{CO} J=6 \rightarrow 5$ and $7 \rightarrow 6$ are about $80^{\prime \prime} \times 60^{\prime \prime}$, but the inner $40^{\prime \prime} \times 40^{\prime \prime}$ region has a better root mean square (rms) noise level than that in the outer region because the integration time was longer in the center. C I $1 \rightarrow 0$ is mapped in a region of size $\sim 15^{\prime \prime} \times 25^{\prime \prime}$.

All observations were performed with frequencies corresponding to a velocity of $v_{\mathrm{LSR}} \sim 420 \mathrm{~km} \mathrm{~s}^{-1}$ (Local Standard of Rest). We list the observation date, instruments, typical rms. noise levels, system temperatures, telescope efficiencies, and map sizes for different epochs in Tables 1 and 2.

\subsection{Spectral line data reduction}

All spectral line data are reduced with the CLASS/GILDAS ${ }^{2}$ package. We inspect each spectrum by eye and classify the spectral quality from baseline flatness and system temperature levels. About $5 \%$ of the spectra are discarded, except for ${ }^{13} \mathrm{CO} \mathrm{J}=3 \rightarrow$ 2 and ${ }^{12} \mathrm{CO} J=7 \rightarrow 6$, for which $50 \%$ and $20 \%$ of the data have to be dropped respectively, because of unstable baselines. Linear baseline is subtracted for each individual spectrum. All spectra are then coadded and resampled to $5 \mathrm{~km} \mathrm{~s}^{-1}$ velocity resolution.

We find that the emission peaks and the intensity distributions of ${ }^{12} \mathrm{CO} J=3 \rightarrow 2,4 \rightarrow 3$, and C I $1 \rightarrow 0$ observed during different epochs have offsets of $\sim 3-5^{\prime \prime}$, which are likely caused by our limited pointing accuracy. These offsets are systematic for each observing epoch. Within the limits of pointing errors $(<1 / 4$ of the beam size), relative intensity distributions are the same for the observed maps. We assume that the central position of Circinus should show particularly strong $\mathrm{CO}$ emission with a

2 http://www.iram.fr/IRAMFR/GILDAS 
Table 1. Parameters of ${ }^{13} \mathrm{CO},{ }^{12} \mathrm{CO}$, and $\mathrm{C}$ I observations

\begin{tabular}{|c|c|c|c|c|c|c|c|c|c|}
\hline Transitions & Date & Receiver & $\eta_{\mathrm{mb}}$ & $\eta_{\mathrm{f}}$ & $\mathrm{Jy} / \mathrm{K}$ & $\begin{array}{c}p w v \\
(\mathrm{~mm})\end{array}$ & $\begin{array}{l}T_{\text {sys }} \\
(\mathrm{K}) \\
\end{array}$ & $\begin{array}{c}\text { Map size } \\
(\mathrm{RA} \times \mathrm{Dec})\end{array}$ & Obs. mode ${ }^{a}$ \\
\hline${ }^{13} \mathrm{CO} J=3 \rightarrow 2$ & $\begin{array}{c}\text { 2006-Jul.-23 } \\
\text { 2009-Oct.-16 }\end{array}$ & $\begin{array}{l}\text { APEX2A } \\
\text { APEX2A }\end{array}$ & $\begin{array}{l}0.73 \\
0.73\end{array}$ & $\begin{array}{l}0.97 \\
0.97\end{array}$ & $\begin{array}{l}40 \\
40 \\
\end{array}$ & $\begin{array}{l}1.1 \\
1.3 \\
\end{array}$ & $\begin{array}{l}190 \cdots 210 \\
500 \cdots 700\end{array}$ & $\begin{array}{l}\text { central point }^{b} \\
\text { central point }\end{array}$ & $\begin{array}{l}\text { ON-OFF } \\
\text { ON-OFF }\end{array}$ \\
\hline${ }^{12} \mathrm{CO} \mathrm{J}=3 \rightarrow 2$ & $\begin{array}{c}\text { 2006-Jun.-27 } \\
\text { 2006-Jul.-23 } \\
\text { 2007-Jun.-30 } \\
\text { 2008-Dec.-26 } \\
\text { 2009-Oct.-16 } \\
\text { 2010-Jul.-14 } \\
\text { 2010-Nov.-15 } \\
\end{array}$ & $\begin{array}{l}\text { APEX2A } \\
\text { APEX2A } \\
\text { APEX2A } \\
\text { APEX2A } \\
\text { APEX2A } \\
\text { FLASH345 } \\
\text { FLASH345 } \\
\end{array}$ & $\begin{array}{l}0.73 \\
0.73 \\
0.73 \\
0.73 \\
0.73 \\
0.73 \\
0.73 \\
\end{array}$ & $\begin{array}{l}0.97 \\
0.97 \\
0.97 \\
0.97 \\
0.97 \\
0.97 \\
0.97 \\
\end{array}$ & $\begin{array}{l}41 \\
41 \\
41 \\
41 \\
41 \\
41 \\
41 \\
\end{array}$ & $\begin{array}{l}1.1 \\
1.1 \\
1.1 \\
1.1 \\
1.1 \\
0.6 \\
1.5 \\
\end{array}$ & $\begin{array}{lll}150 & \cdots & 160 \\
180 & \cdots & 220 \\
230 & \cdots & 250 \\
300 & \cdots & 345 \\
440 & \cdots & 450 \\
190 & \cdots & 210 \\
350 & \cdots & 380 \\
\end{array}$ & $\begin{array}{c}\text { central point } \\
40^{\prime \prime} \times 40^{\prime \prime} \\
40^{\prime \prime} \times 25^{\prime \prime} \\
\text { central point } \\
\text { central point } \\
20^{\prime \prime} \times 30^{\prime \prime} \\
\text { central point }\end{array}$ & $\begin{array}{l}\text { ON-OFF } \\
\text { RASTER } \\
\text { RASTER } \\
\text { ON-OFF } \\
\text { ON-OFF } \\
\text { RASTER } \\
\text { ON-OFF } \\
\end{array}$ \\
\hline${ }^{12} \mathrm{CO} \mathrm{J}=4 \rightarrow 3$ & 2009-Jun.-07 & FLASH460 & 0.60 & 0.95 & 48 & 0.60 & $500 \cdots 600$ & $30^{\prime \prime} \times 40^{\prime \prime}$ & RASTER \\
\hline${ }^{12} \mathrm{CO} J=6 \rightarrow 5$ & $\begin{array}{l}\text { 2009-Jun.-13 } \\
\text { 2009-Aug.-10 }\end{array}$ & $\begin{array}{l}\text { CHAMP }^{+}-\mathrm{I} \\
\text { CHAMP }^{+} \text {-I }\end{array}$ & $\begin{array}{l}0.52 \\
0.52 \\
\end{array}$ & $\begin{array}{l}0.95 \\
0.95\end{array}$ & $\begin{array}{l}53 \\
53\end{array}$ & $\begin{array}{l}0.52 \\
0.27 \\
\end{array}$ & $\begin{array}{lll}1500 & \cdots & 2100 \\
1000 & \cdots & 1300 \\
\end{array}$ & $\begin{array}{l}80^{\prime \prime} \times 60^{\prime \prime} \\
80^{\prime \prime} \times 60^{\prime \prime}\end{array}$ & $\begin{array}{l}\text { OTF } \\
\text { OTF }\end{array}$ \\
\hline${ }^{12} \mathrm{CO} J=7 \rightarrow 6$ & $\begin{array}{l}\text { 2009-Jun.-13 } \\
\text { 2009-Aug.-10 }\end{array}$ & $\begin{array}{l}\text { CHAMP }^{+} \text {-II } \\
\text { CHAMP }^{+} \text {-II } \\
\end{array}$ & $\begin{array}{l}0.49 \\
0.49 \\
\end{array}$ & $\begin{array}{l}0.95 \\
0.95 \\
\end{array}$ & $\begin{array}{l}70 \\
70 \\
\end{array}$ & $\begin{array}{l}0.49 \\
0.49 \\
\end{array}$ & $\begin{array}{lll}4000 & \cdots & 9000 \\
2500 & \cdots & 4000 \\
\end{array}$ & $\begin{array}{l}80^{\prime \prime} \times 60^{\prime \prime} \\
80^{\prime \prime} \times 60^{\prime \prime}\end{array}$ & $\begin{array}{l}\text { OTF } \\
\text { OTF }\end{array}$ \\
\hline $\mathrm{C}$ I $1 \rightarrow 0$ & 2010-Jul.-14 & FLASH460 & 0.60 & 0.95 & 50 & 0.60 & $700 \cdots 1000$ & $15^{\prime \prime} \times 25^{\prime \prime}$ & ON-OFF \\
\hline
\end{tabular}

Notes. ${ }^{(a)}$ ON-OFF: position switching; RASTER: raster scan; OTF: On-The-Fly scan. ${ }^{(b)}$ We adopt $\alpha(J 2000)=14^{\mathrm{h}} 13^{\mathrm{m}} 10.0^{\mathrm{s}}, \delta(J 2000)=$ $-65^{\circ} 20^{\prime} 21^{\prime \prime} .0$ as the central position of Circinus.

Table 2. Parameters of the observed lines.

\begin{tabular}{cccc}
\hline \hline Transitions & $\begin{array}{c}v_{\text {rest }} \\
(\mathrm{GHz})\end{array}$ & $\begin{array}{c}\text { Resolution } \\
\left({ }^{\prime \prime}\right)\end{array}$ & $\begin{array}{c}\mathrm{rms}^{a} \\
(\mathrm{~K})\end{array}$ \\
\hline${ }^{13} \mathrm{CO} J=3 \rightarrow 2$ & 330.588 & 19.0 & 0.02 \\
${ }^{12} \mathrm{CO} J=3 \rightarrow 2$ & 345.796 & 18.2 & 0.06 \\
${ }^{12} \mathrm{CO} J=4 \rightarrow 3$ & 461.041 & 14.0 & 0.1 \\
${ }^{12} \mathrm{CO} J=6 \rightarrow 5$ & 691.473 & 9.4 & 0.3 \\
${ }^{12} \mathrm{CO} J=7 \rightarrow 6$ & 806.652 & 8.2 & 1.0 \\
$\mathrm{C} \mathrm{I} 1 \rightarrow 0$ & 492.161 & 13.5 & 0.12 \\
\hline
\end{tabular}

Notes. ${ }^{(a)} 1 \sigma$ noise level in units of $T_{\mathrm{mb}}$ calculated from the datacubes with a channel width of $5 \mathrm{~km} \mathrm{~s}^{-1}$.

symmetrical line profile, and this profile should also correspond to the peak position of the integrated intensity in an individual map. We fit the map distributions and shift the positions of ${ }^{12} \mathrm{CO} J=3 \rightarrow 2,4 \rightarrow 3$, and $\mathrm{CI} 1 \rightarrow 0$ accordingly, and then coadd the maps to improve signal-to-noise ratios $(\mathrm{S} / \mathrm{N})$. From several measurement epochs, we estimate calibration uncertainties to be $10 \%$ for ${ }^{12} \mathrm{COJ}=3 \rightarrow 2$ and $4 \rightarrow 3$, and $15 \%$ for ${ }^{12} \mathrm{CO} J=6 \rightarrow 5$ and $7 \rightarrow 6$.

We convert the antenna temperature $\left(T_{\mathrm{A}}^{\star}\right)$ to the main beam brightness temperature $\left(T_{\mathrm{mb}}\right)$ scale, using $T_{\mathrm{mb}}=T_{\mathrm{A}}^{\star} \cdot \eta_{\mathrm{f}} / B_{\mathrm{eff}}$, where $\eta_{\mathrm{f}}$ and $B_{\text {eff }}$ are the forward hemisphere and beam efficiency of the telescope. We list them in Table 1. All spectra presented in this paper are in units of $T_{\mathrm{mb}}(\mathrm{K})$. In Table 2 we list frequencies, angular resolutions, and noise levels of the reduced data.

For each transition, we combine all the calibrated spectra and use the gridding routine XY_MAP in CLASS to construct datacubes, with weightings proportional to $1 / \sigma^{2}$, where $\sigma$ is the rms. noise level. This routine convolves the gridded data with a Gaussian kernel of full width to half maximum (FWHM) $\sim 1 / 3$ the telescope beam size, yielding a final angular resolution slightly coarser than the original beam size. For the analysis below, we further convolve the datacubes to several angular resolutions to facilitate comparisons with data from the literature.

\subsection{Other archival data}

We obtained a $70 \mu \mathrm{m}$ image observed with the Photoconductor Array Camera and Spectrometer (PACS) on board the Herschel space telescope ${ }^{3}$ through the Herschel Science Archive. We downloaded the post processed data of level 2.5. The observation ID is 1342203269 , and it contains data observed on 20 August 2010. We also used an archival $\mathrm{H} \alpha$ image of the Hubble Space Telescope (HST; Wilson et al. 2000), downloaded from the NASA/IPAC Extragalactic Database (NED).

\section{Results}

\subsection{Spatial distributions}

\subsubsection{Herschel $70 \mu \mathrm{m}$ and HST maps of the Circinus galaxy}

The left panel of Fig. 1 shows Herschel $70 \mu \mathrm{m}$ contours overlaid on an HST H $\alpha$ image (Wilson et al. 2000). The $70 \mu \mathrm{m}$ emission has a concentration in the very central region, and an extended emission out to about 40" (in diameter). The $\mathrm{H} \alpha$ emission shows structures of both the S-F ring and the central nuclear region. Therefore, we separate the center of Circinus into three regions: the nuclear region $\left(D<18^{\prime \prime} \sim 360 \mathrm{pc}\right)$, the entire central $45^{\prime \prime}$ $\left(D<45^{\prime \prime} \sim 900 \mathrm{pc}\right)$ region, and the S-F ring region $\left(18^{\prime \prime}<\right.$ $\left.D<45^{\prime \prime}\right)$. We define the $\mathrm{H} \alpha$ bright ring like structure as the S-F ring, rather than the ring structure modeled in Curran et al. (1999). The concentric circles show the nuclear region (white) and the entire central region (red) including both the nucleus and the S-F ring. The peak of the $70 \mu \mathrm{m}$ emission is consistent with the center of the CO emission (e.g., Curran et al. 2001), and has a slight shift down to the south of the $\mathrm{H} \alpha$ peak, which is likely attenuated by the dust extinction. The central $18^{\prime \prime}$ region contributes $\sim 20 \%$ of the $70 \mu \mathrm{m}$ emission of the entire galaxy. These features are also consistent with the Spitzer mid-infrared images (For et al. 2012).

3 Herschel is an ESA space observatory with science instruments provided by European-led Principal Investigator consortia and with important participation from NASA. 

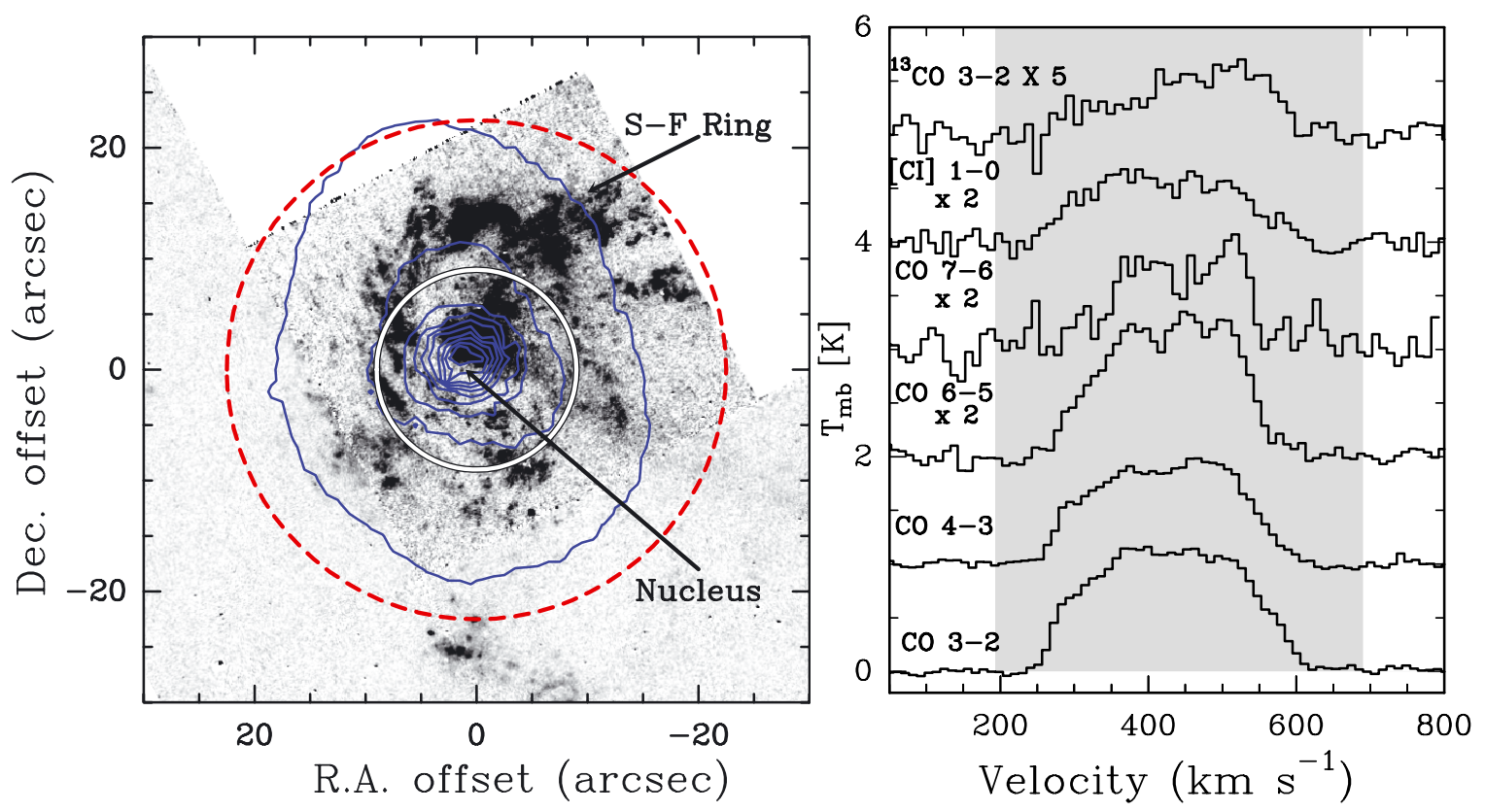

Fig. 1. Left panel: Herschel $70 \mu \mathrm{m}$ contours overlaid on an $\mathrm{H} \alpha$ image of the HST (Wilson et al. 2000). The contour levels are 500, 1000, 2000, 3000 , and $4000 \mathrm{MJy} / \mathrm{sr}$. The concentric circles show the beam sizes of ${ }^{12} \mathrm{CO} J=3 \rightarrow 2$ for $\operatorname{APEX}\left(18^{\prime \prime}\right.$, white thick line) and ${ }^{12} \mathrm{CO} J=1 \rightarrow 0$ for SEST (45", red dashed line). Right panel: CO spectra observed in the central position. ${ }^{12} \mathrm{CO} J=6 \rightarrow 5,7 \rightarrow 6$, and C I are multiplied by a factor of 2 , and ${ }^{13} \mathrm{CO} J=3 \rightarrow 2$ is multiplied by a factor of 5 . The (shaded) line emission ranges from $\sim 200$ to $\sim 700 \mathrm{~km} \mathrm{~s}^{-1}$.

\subsection{2. $\mathrm{CO}$ and $\mathrm{CI} 1 \rightarrow 0$ spectra}

The CO and C I spectra from the central position (Fig. 1, right panel) are shown with their original angular resolutions (before the convolution in XY_MAP). Although the ${ }^{12} \mathrm{CO} J=7 \rightarrow 6$ and $\mathrm{C}$ I lines have relatively low $\mathrm{S} / \mathrm{N}$, all of the line profiles look fairly similar, i.e., their intensity ratios are constant to within $30 \%$ as a function of velocity. This implies that overall, the gas components probed by the different lines follow the same kinematics.

We convolve Gaussian kernels with all datacubes to match the angular resolutions of the low-J CO data. Using the beam matched datacubes, we extract spectra in the central position and calculate the integrated line intensities in the velocity range from $200 \mathrm{~km} \mathrm{~s}^{-1}$ to $700 \mathrm{~km} \mathrm{~s}^{-1}$. Table 3 summarizes the observed line properties at the angular resolutions of $18^{\prime \prime}$ and $45^{\prime \prime}$.

Figure 2 shows spectra of $\mathrm{CI}$ and ${ }^{12} \mathrm{CO} J=3 \rightarrow 2$ from the central region of Circinus. We obtained these two lines simultaneously, free from pointing inaccuracy. We overlay their spectra in their original angular resolutions, $\sim 12.5^{\prime \prime}$ for $\mathrm{C}$ I and $\sim 18^{\prime \prime}$ for ${ }^{12} \mathrm{CO} J=3 \rightarrow 2$. At most positions, there is no obvious discrepancy between the line profiles of ${ }^{12} \mathrm{COJ}=3 \rightarrow 2$ and $\mathrm{CI}$, neither in the central position nor at the edges of the mapped region.

\subsection{3. $\mathrm{CO}$ and $\mathrm{CI}$ maps}

Figure 3 presents the integrated intensity images (moment-zero maps) of all ${ }^{12} \mathrm{CO}$ transitions mapped with the APEX telescope. The dotted thick (red) contour lines present half of the peak intensity level for each map. The $\mathrm{CO}$ emission of all transitions is well confined within the central $40^{\prime \prime} \times 40^{\prime \prime}$ region. To increase the $\mathrm{S} / \mathrm{N}$ level of ${ }^{12} \mathrm{CO} J=7 \rightarrow 6$, we convolved it to the angular resolution of the ${ }^{12} \mathrm{CO} J=3 \rightarrow 2$ map. The ${ }^{12} \mathrm{CO} J=6 \rightarrow 5$ and $J=7 \rightarrow 6$ distributions show elongations along the major axis, i.e., along the direction from the northeast to the southwest. The thin dotted contours (blue) in the ${ }^{12} \mathrm{CO} J=6 \rightarrow 5$ and

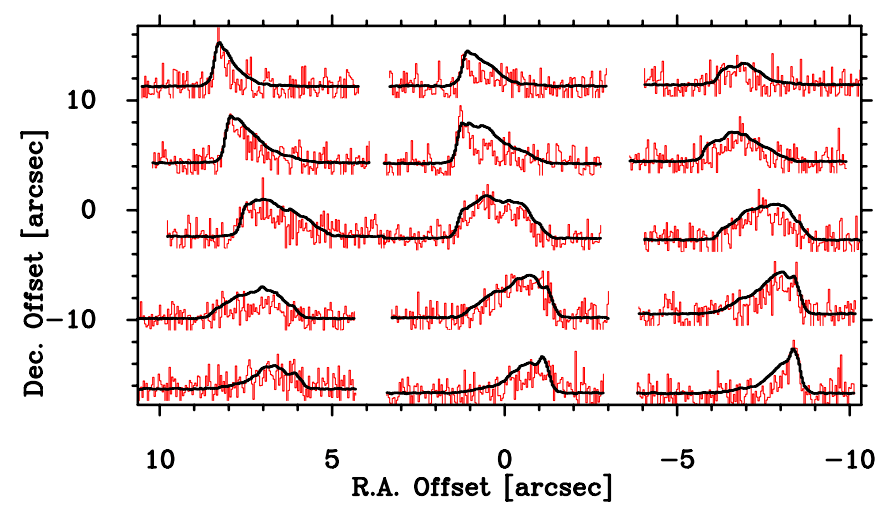

Fig. 2. C I $1 \rightarrow 0$ and ${ }^{12} \mathrm{CO} J=3 \rightarrow 2$ spectra from the central region of the Circinus galaxy. The CI spectra are relatively noisier and are presented in red, while the ${ }^{12} \mathrm{CO} J=3 \rightarrow 2$ profiles are plotted in black. To reach similar intensities, $\mathrm{C}$ I is scaled up by a factor of three.

$J=7 \rightarrow 6$ maps mark the regions with almost uniform scanning coverage in the OTF mappings, so these regions have lower noise levels than those outside. We mask the corners of the ${ }^{12} \mathrm{CO} J=7 \rightarrow 6$ image to avoid displaying regions farther out with high noise and poor baselines.

To explore the sizes of the emitting regions in different $\mathrm{CO}$ transitions, we deconvolve the moment-zero maps with circular Gaussian beams, and fit the source sizes using two-dimensional Gaussian models. For ${ }^{12} \mathrm{CO} J=3 \rightarrow 2,4 \rightarrow 3$, and $6 \rightarrow 5$, the beam sizes (FWHM) of 18", 14.0", and 9.4" (see Table 2) are adopted to deconvolve the images, respectively. The $\mathrm{S} / \mathrm{N}$ of the ${ }^{12} \mathrm{CO} J=7 \rightarrow 6$ map is not high enough to provide reliable fitting results. We list the fitting parameters in Table 4. For ${ }^{12} \mathrm{CO} J=3 \rightarrow 2$ we get a position angle of $33.5^{\circ}$, which is adopted in the following analysis to define the major axis of molecular gas emission. This result is also close to the position angle of $34^{\circ}$ derived from ${ }^{12} \mathrm{COJ}=1 \rightarrow 0$ and $2 \rightarrow 1$ maps (Curran et al. 2008). The fitted position angle of C I $1 \rightarrow 0$ 
Table 3. CO line intensities.

\begin{tabular}{|c|c|c|c|c|c|c|}
\hline \multirow[t]{2}{*}{ Transitions } & \multicolumn{3}{|c|}{ Beam $=18^{\prime \prime}$} & \multicolumn{3}{|c|}{ Beam $=45^{\prime \prime}$} \\
\hline & $\begin{array}{r}W_{\text {line }}{ }^{a} \\
\mathrm{Kkm} \mathrm{s}^{-1} \\
\end{array}$ & $\begin{array}{l}F_{\text {line }} \\
\mathrm{Jy} \mathrm{km} \mathrm{s}^{-1}\end{array}$ & $\begin{array}{l}L_{\text {line }}^{\prime} / L_{\mathrm{CO} 3-2}^{\prime} \\
-\end{array}$ & $\begin{array}{c}W_{\text {line }} \\
\mathrm{Kkm} \mathrm{s}^{-1} \\
\end{array}$ & $\begin{array}{c}F_{\text {line }} \\
\mathrm{Jy} \mathrm{km} \mathrm{s}^{-1} \\
\end{array}$ & $\begin{array}{c}L_{\text {line }}^{\prime} / L_{\mathrm{CO} 3-2}^{\prime} \\
-\end{array}$ \\
\hline${ }^{12} \mathrm{CO} J=1 \rightarrow 0$ & - & - & - & $180 \pm 35^{b}$ & $3.51 \times 10^{3}$ & 1 \\
\hline${ }^{12} \mathrm{CO} J=2 \rightarrow 1$ & - & - & - & $144 \pm 25^{b}$ & $1.42 \times 10^{4}$ & $0.83 \pm 0.20$ \\
\hline${ }^{12} \mathrm{CO} \mathrm{J}=3 \rightarrow 2$ & $310 \pm 20$ & $0.97 \times 10^{4}$ & 1 & $140 \pm 20$ & $2.49 \times 10^{4}$ & $0.79 \pm 0.16$ \\
\hline${ }^{12} \mathrm{CO} J=4 \rightarrow 3$ & $240 \pm 20$ & $1.17 \times 10^{4}$ & $0.77 \pm 0.2$ & $80 \pm 15$ & $2.51 \times 10^{4}$ & $0.44 \pm 0.09$ \\
\hline${ }^{12} \mathrm{CO} J=6 \rightarrow 5$ & $140 \pm 15$ & $1.44 \times 10^{4}$ & $0.45 \pm 0.08$ & $35 \pm 5$ & $2.48 \times 10^{4}$ & $0.19 \pm 0.03$ \\
\hline${ }^{12} \mathrm{CO} J=7 \rightarrow 6$ & $55 \pm 7$ & $0.97 \times 10^{4}$ & $0.18 \pm 0.03$ & $19 \pm 5$ & $1.73 \times 10^{4}$ & $0.11 \pm 0.03$ \\
\hline${ }^{13} \mathrm{CO} J=1 \rightarrow 0$ & - & - & - & $13 \pm 2.5$ & 230 & $0.07 \pm 0.02$ \\
\hline${ }^{13} \mathrm{CO} J=2 \rightarrow 1$ & - & - & - & $12.5 \pm 2.5$ & 1300 & $0.07 \pm 0.02$ \\
\hline${ }^{13} \mathrm{CO} J=3 \rightarrow 2$ & $24 \pm 5$ & 740 & $0.07 \pm 0.02$ & $9.5 \pm 3.0^{c}$ & 1850 & $0.05 \pm 0.02$ \\
\hline $\mathrm{C}_{\mathrm{I}} 1 \rightarrow 0$ & $100 \pm 25$ & $5 \times 10^{3}$ & & $45 \pm 10$ & $1.4 \times 10^{4}$ & \\
\hline
\end{tabular}

Notes. ${ }^{(a)}$ The integrated line intensities are calculated from $W_{\text {line }}=\int T_{\mathrm{mb}} \mathrm{d} v$ in the velocity range from $200 \mathrm{~km} \mathrm{~s}^{-1}$ to $700 \mathrm{~km} \mathrm{~s}^{-1}$. ${ }^{(b)}$ We take aperture efficiencies of $\eta_{\mathrm{mb}}=0.7$ and 0.6 for ${ }^{12} \mathrm{CO} J=1 \rightarrow 0$ and ${ }^{12} \mathrm{CO} J=2 \rightarrow 1$ for SEST. ${ }^{(c)}$ We convolve the ${ }^{13} \mathrm{CO} J=3 \rightarrow 2$ emission to a resolution of $45^{\prime \prime}$, assuming that the distribution of ${ }^{13} \mathrm{CO} J=3 \rightarrow 2$ is the same as that of ${ }^{12} \mathrm{CO} J=3 \rightarrow 2$.
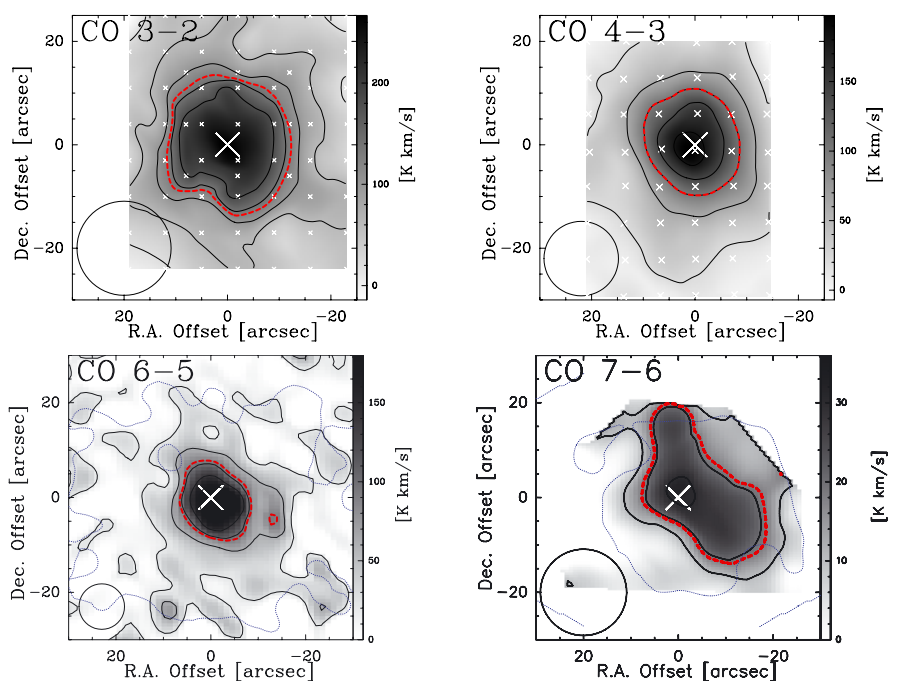

Fig. 3. Moment-zero images of multiple- $J$ CO transitions. Big crosses mark the central position of Circinus; small crosses in the upper panels denote the sampled positions. Upper left: ${ }^{12} \mathrm{CO} J=3 \rightarrow 2$; upper right: ${ }^{12} \mathrm{CO} \mathrm{J}=4 \rightarrow 3$; lower left: ${ }^{12} \mathrm{COJ}=6 \rightarrow 5$; lower right: ${ }^{12} \mathrm{CO} \mathrm{J}=$ $7 \rightarrow 6$. Circles in the lower left of each panel show the beam. The ${ }^{12} \mathrm{CO} J=7 \rightarrow 6$ map was convolved to an angular resolution of $18^{\prime \prime}$. Contour levels are $20,60, \ldots, 180 \mathrm{~K} \mathrm{~km} \mathrm{~s}^{-1}$ in steps of $40 \mathrm{~K} \mathrm{~km} \mathrm{~s}^{-1}$ for ${ }^{12} \mathrm{CO} J=3 \rightarrow 2, J=4 \rightarrow 3$, and $J=6 \rightarrow 5$, and $10,20,30 \mathrm{~K} \mathrm{~km} \mathrm{~s}^{-1}$ for ${ }^{12} \mathrm{CO} J=7 \rightarrow 6\left(1 \sigma=1.9,3,10\right.$, and $3 \mathrm{~K} \mathrm{~km} \mathrm{~s}^{-1}$ for ${ }^{12} \mathrm{CO} J=$ $3 \rightarrow 2, J=4 \rightarrow 3, J=6 \rightarrow 5$, and $J=7 \rightarrow 6$ ). Red (thick dotted) contours present the half maximum level of all images. The thin dotted lines in the lower two panels denote the regions that have been scanned with higher $\mathrm{S} / \mathrm{N}$ than other regions farther away from the centers (see Sects. 2 and 3.1.3). The outer dotted lines in the ${ }^{12} \mathrm{CO} J=7 \rightarrow 6$ map are related to masking.

is $65.5^{\circ}$, which is much larger than those determined from the $\mathrm{CO}$ images. This is most likely a consequence of the small size of our CI map. Because C I emission follows CO in all studied cases (e.g., Ikeda et al. 2002; Zhang et al. 2007), a different distribution is highly unlikely.

In Fig. 4, we present the integrated intensity image of C I $492 \mathrm{GHz}$ emission. In spite of a smaller mapping area compared to $\mathrm{CO}$, the thick dotted (red) line denoting the half maximum level of the emission peak is still mostly within the confines

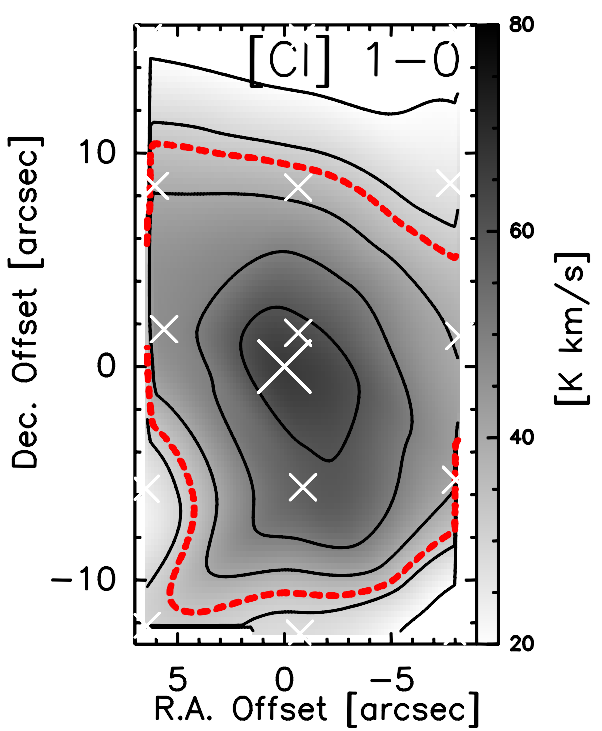

Fig. 4. The $492 \mathrm{GHz}$ C I $1 \rightarrow 0$ integrated intensity image of the central part of the Circinus galaxy. Plotted contour levels are: $20(6 \sigma), 30,40$, 50 , and $60 \mathrm{mK} \mathrm{km} \mathrm{s}^{-1}$. The red contour presents the half maximum level of the C I emission. The beam size (FWHM) is 13.5".

of the map. The detected structure covers an angular distance of $\sim 20^{\prime \prime}$ from northeast to southwest, corresponding to $400 \mathrm{pc}$ on the linear scale. Both the nuclear region and the S-F ring seen in the ${ }^{12} \mathrm{CO} J=1 \rightarrow 0$ and $2 \rightarrow 1$ images (Curran et al. 1998) are covered by the $\mathrm{C}$ I $1 \rightarrow 0$ map.

\subsection{Gas kinematics}

\subsubsection{CO channel maps}

In Fig. 5, we plot the channel maps of ${ }^{12} \mathrm{CO} J=3 \rightarrow 2,4 \rightarrow 3$, and $6 \rightarrow 5$. The northeastern side of Circinus is approaching and the southwestern side is receding. ${ }^{12} \mathrm{CO} J=6 \rightarrow 5$ is highly concentrated near the peaks of the ${ }^{12} \mathrm{CO} J=3 \rightarrow 2$ and $4 \rightarrow 3$, i.e., near the central position of Circinus. The systematic velocity variations of different $\mathrm{CO}$ transitions are apparent. The emission of all three lines is particularly strong at the velocity bins of $300-400 \mathrm{~km} \mathrm{~s}^{-1}$ and $450-550 \mathrm{~km} \mathrm{~s}^{-1}$, and the brightness 
Table 4. Fitting parameters of CO moment-zero maps.

\begin{tabular}{lllllc}
\hline \hline & \multicolumn{3}{c}{ Apparent size } & \multicolumn{3}{c}{ Deconvolved size } \\
\hline Line & $\begin{array}{l}\text { Major axis } \\
\left({ }^{\prime \prime}\right)\end{array}$ & $\begin{array}{l}\text { Minor axis } \\
\left({ }^{\prime \prime}\right)\end{array}$ & $\begin{array}{l}\text { Major axis } \\
\left({ }^{\prime \prime}\right)\end{array}$ & $\begin{array}{c}\text { Minor axis } \\
\left({ }^{\prime \prime}\right)\end{array}$ & $\begin{array}{c}\text { Pos. angle } \\
\left({ }^{\circ}\right)\end{array}$ \\
\hline${ }^{12} \mathrm{CO} J=3 \rightarrow 2$ & $28.1 \pm 0.4$ & $27.1 \pm 0.4$ & $20.6 \pm 0.4$ & $19.1 \pm 0.4$ & 33.5 \\
${ }^{12} \mathrm{CO} J=4 \rightarrow 3$ & $24.0 \pm 0.5$ & $21.4 \pm 0.5$ & $19.2 \pm 0.5$ & $15.8 \pm 0.4$ & 36.0 \\
${ }^{12} \mathrm{CO} J=6 \rightarrow 5$ & $17.6 \pm 0.5$ & $14.2 \pm 0.5$ & $14.8 \pm 0.6$ & $10.5 \pm 0.5$ & 40.0 \\
$\mathrm{CI} 1 \rightarrow 0^{a}$ & $21.4 \pm 3.2$ & $14.4 \pm 0.9$ & $16.7 \pm 3.0$ & $5.2 \pm 1.0$ & 65.5 \\
\hline
\end{tabular}

Notes. ${ }^{(a)}$ The fitted size of the minor axis and the position angle could be affected by incomplete mapping. Curran et al. (2008) obtain $34^{\circ} \pm 4^{\circ}$ as the large scale position angle from $\mathrm{CO} J=1 \rightarrow 0$ and $2 \rightarrow 1$ data.

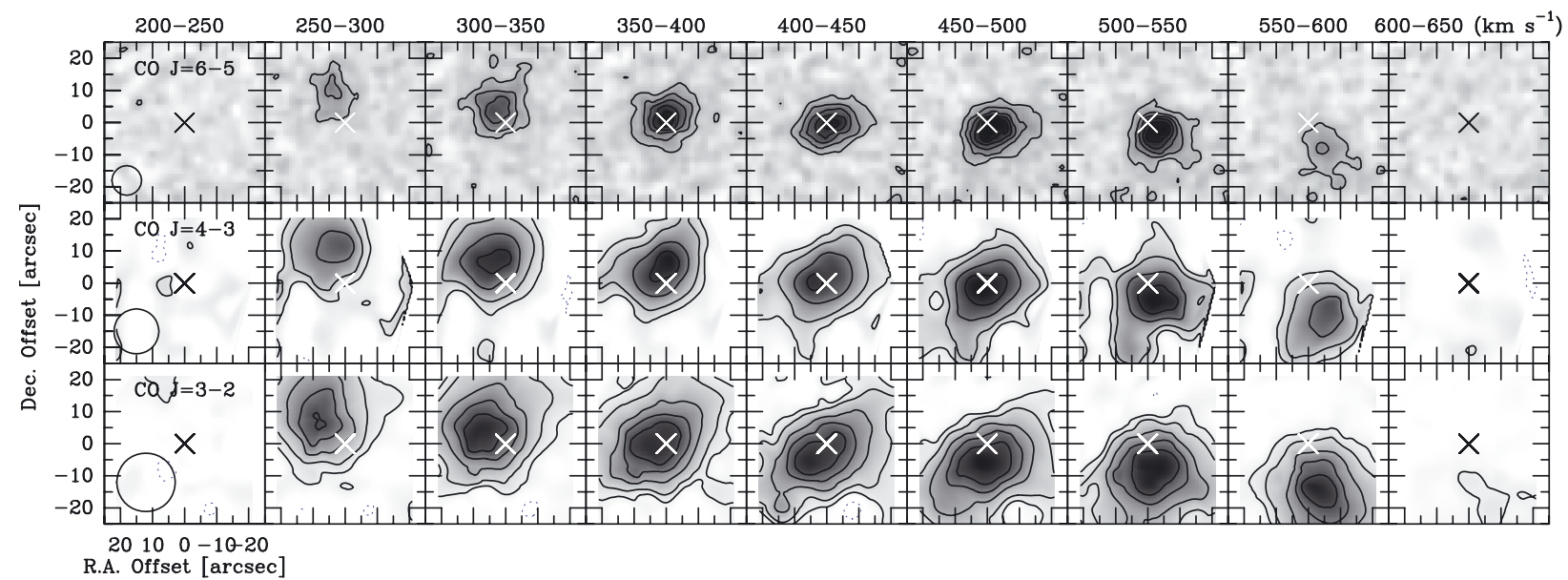

Fig. 5. CO channel maps of the central region of the Circinus galaxy. The velocity range is given at the top, and the nuclear position of the Circinus galaxy is labeled by white crosses. Beam sizes are shown in the panels at the left hand side. Upper panels: ${ }^{12} \mathrm{CO} J=6 \rightarrow 5$ channel maps. The contours are from $0.1(2 \sigma)$ to $0.5 \mathrm{~K}$ with a spacing of $0.1 \mathrm{~K}$. Middle panels: ${ }^{12} \mathrm{CO} J=4 \rightarrow 3$ channel maps. The contours are -0.1 (dotted) and 0.1 to $1.0 \mathrm{~K} \mathrm{~km} \mathrm{~s}^{-1}$, the latter with a spacing of $0.1 \mathrm{~K}$. The $3 \sigma$ noise level corresponds to $0.1 \mathrm{~K}$. Lower panels: ${ }^{12} \mathrm{CO} J=3 \rightarrow 2$ channel maps. The contours are the same as for ${ }^{12} \mathrm{CO} J=4 \rightarrow 3$. The $5 \sigma$ noise level corresponds to $0.1 \mathrm{~K}$.

temperature peaks even exceed those in the central velocity bin ranging from 400 to $450 \mathrm{~km} \mathrm{~s}^{-1}$. The ${ }^{12} \mathrm{CO} J=4 \rightarrow 3$ and $6 \rightarrow 5$ emission drops faster than that of ${ }^{12} \mathrm{CO} J=3 \rightarrow 2$ when the velocity is higher than $550 \mathrm{~km} \mathrm{~s}^{-1}$ and lower than $300 \mathrm{~km} \mathrm{~s}^{-1}$.

\subsubsection{CO P-V diagrams}

Figure 6 shows the position-velocity (P-V) diagrams of ${ }^{12} \mathrm{CO} J=3 \rightarrow 2,4 \rightarrow 3$, and $6 \rightarrow 5$, along the major axis of Circinus. We make cuts with a position angle of $33.5^{\circ}$, which is from the fitting result of Sect. 3.1.3 (see also Table 4). In the $\mathrm{P}-\mathrm{V}$ diagram of ${ }^{12} \mathrm{CO} J=3 \rightarrow 2$, the ridge of maximum intensity covers a velocity range of about $400 \mathrm{~km} \mathrm{~s}^{-1}$, in accordance with the high inclination $\left(i \sim 65-78^{\circ}\right.$; Curran et al. 2008) of the galaxy, over a region of roughly $\pm 10^{\prime \prime}$. The rotation field in this area is characterized by a velocity gradient of $\mathrm{d} v / \mathrm{d} \theta=$ $400 \mathrm{~km} \mathrm{~s}^{-1} / 20^{\prime \prime}\left(\sim 20 \mathrm{~km} \mathrm{~s}^{-1} / \operatorname{arcsec}\right)$, corresponding to $\mathrm{d} v / \mathrm{d} r \sim$ $1.0 \mathrm{~km} \mathrm{~s}^{-1} \mathrm{pc}^{-1}$ in the plane of the galaxy, when an inclination angle of $65^{\circ}$ is adopted (Freeman et al. 1977). The higher- $J$ level and the higher the angular resolution, the steeper the rotation curve appears. The ${ }^{12} \mathrm{CO} J=4 \rightarrow 3$ distribution looks similar to that of ${ }^{12} \mathrm{CO} J=3 \rightarrow 2$, but appears to be slimmer because of the higher angular resolution. For ${ }^{12} \mathrm{CO} J=6 \rightarrow 5$, the ridge of maximum intensity covers a velocity range of $\sim 350 \mathrm{~km} \mathrm{~s}^{-1}$ in a small region encompassing offsets of $\pm 5^{\prime \prime}$. We derive a velocity gradient of $\sim 350 \mathrm{~km} \mathrm{~s}^{-1} / 10^{\prime \prime}\left(\mathrm{d} v / \mathrm{d} r \sim 1.5 \mathrm{~km} \mathrm{~s}^{-1} \mathrm{pc}^{-1}\right)$, which corresponds to $\mathrm{d} v / \mathrm{d} r \sim 1.7 \mathrm{~km} \mathrm{~s}^{-1} \mathrm{pc}^{-1}$ when an inclination angle of $65^{\circ}$ is applied. Limited by the spatial resolution, these velocity gradients only provide lower limits for the actual rotational motions in the central region of Circinus.

We estimate the dynamical mass from $M_{\text {dyn }}\left[M_{\odot}\right]=230 \times$ $v_{\text {rot }}^{2}\left[\mathrm{~km} \mathrm{~s}^{-1}\right] \times r$ [pc] (e.g., Schinnerer et al. 2000), where $v_{\text {rot }}$ is the inclination-corrected rotation speed in $\mathrm{km} \mathrm{s}^{-1}$, and $r$ is the radius in pc. We find a rotation velocity $v_{\text {rot }}=\left(340 \mathrm{~km} \mathrm{~s}^{-1} / 2\right) / \sin \left(65^{\circ} \pm\right.$ $\left.5^{\circ}\right) \sim 190 \pm 10 \mathrm{~km} \mathrm{~s}^{-1}$, and derive dynamic masses of $M_{\text {dyn }}=$ $1.4 \pm 0.1 \times 10^{9} M_{\odot}$ within $180 \mathrm{pc}$ of the center, and $3.6 \pm 0.4 \times$ $10^{9} M_{\odot}$ within a galactocentric radius of $450 \mathrm{pc}$. The latter is consistent with the dynamical mass of $3.3 \pm 0.3 \times 10^{9} M_{\odot}$ estimated for an outer radius of $560 \mathrm{pc}$ by Curran et al. (1998).

Unlike the case of the moment-zero maps, where all $\mathrm{CO}$ emission peaks at the central position, the $\mathrm{CO}$ transitions in the P-V diagrams mainly peak at the edges of the velocity distributions on the major axis. This scenario indicates a central molecular void and a circumnuclear ring. The P-V diagram of ${ }^{12} \mathrm{CO} J=3 \rightarrow 2$ is, within the errors, symmetric for the lowest emission levels around the AGN, with respect to a radial velocity of $430 \pm 20 \mathrm{~km} \mathrm{~s}^{-1}$. The earlier adopted central positions (Figs. 2-6) are consistent with the dynamical center of the galaxy and the velocity can be interpreted as the systemic velocity $\left(v_{\text {sys }}\right)$. 

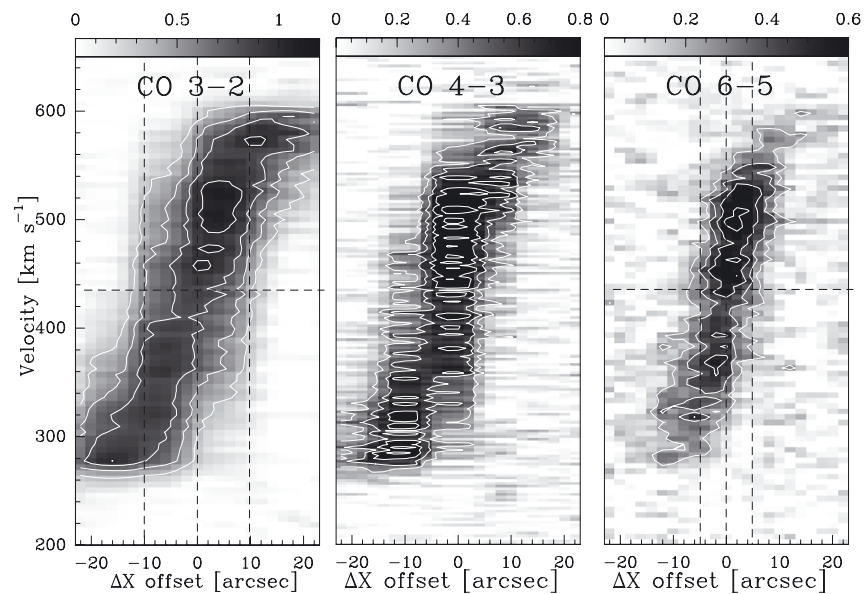

Fig. 6. Position-velocity (P-V) maps of $\mathrm{CO}$ emission from the Circinus galaxy. From left to right: $\mathrm{P}-\mathrm{V}$ diagrams of ${ }^{12} \mathrm{CO} J=3 \rightarrow 2,{ }^{12} \mathrm{CO} J=$ $4 \rightarrow 3$, and ${ }^{12} \mathrm{CO} J=6 \rightarrow 5$. The slice is taken along the major axis obtained from ${ }^{12} \mathrm{CO} J=3 \rightarrow 2$ (position angle: 33.5 , see Table 4 ) and the direction is from northeast (bottom) to southwest (top). The contours are from $30 \%$ to $90 \%$ of the peak intensities with a spacing of $20 \%$.

\section{Excitation conditions and discussions}

Including our new measurements, ${ }^{12} \mathrm{CO}$ has been observed toward the nuclear region of Circinus in all transitions up to $J=7 \rightarrow 6$, except for $J=5 \rightarrow 4$ (e.g., Aalto et al. 1991; Israel 1992; Elmouttie et al. 1997; Curran et al. 1998, 2001; Hitschfeld et al. 2008). The rare isotopologue ${ }^{13} \mathrm{CO}$ has been measured in transitions from $J=1 \rightarrow 0$ to $J=2 \rightarrow 1$ (e.g., Curran et al. 1998, 2001; Hitschfeld et al. 2008), and $J=3 \rightarrow 2$ (this paper). The low- $J$ transitions were observed mostly with SEST $15 \mathrm{~m}$ and Mopra $22 \mathrm{~m}$ (equivalent $15 \mathrm{~m}$ beam size at 115 $\mathrm{GHz}$; Elmouttie et al. 1997), while the mid-J transitions were observed with the APEX $12 \mathrm{~m}$ and the NANTEN-2 $4 \mathrm{~m}$ telescopes. Table 3 summarizes ${ }^{12} \mathrm{CO}$ and ${ }^{13} \mathrm{CO}$ observations collected from the literature.

The wide range of critical density ${ }^{4}$ (from $\sim 3 \times 10^{2} \mathrm{~cm}^{-3}$ for $\mathrm{CO} J=1 \rightarrow 0$ to $\sim 4 \times 10^{5} \mathrm{~cm}^{-3}$ for $\mathrm{CO} J=7 \rightarrow 6$ ) and $E_{\text {upper }} / k_{\mathrm{B}}$ (from $5.5 \mathrm{~K}$ to $155 \mathrm{~K}$ ) of CO lines from $J=1 \rightarrow 0$ up to $J=7 \rightarrow 6$ allows us to probe the molecular gas physical conditions ranging from the cold and low-density average states in giant molecular clouds all the way up to the state of the gas found only near their S-F regions (e.g., Yang et al. 2010; Bradford et al. 2005). Bright ${ }^{12} \mathrm{CO} J=6 \rightarrow 5$ and $J=7 \rightarrow 6$ emission in Circinus implies that there is a large amount of molecular gas in a highly excited phase, while the low- $J$ CO transition lines are also sensitive to the colder and possibly more diffuse gas phase.

\subsection{The large velocity gradient radiative transfer model}

To estimate the physical parameters of the molecular gas, we employ a large velocity gradient (LVG) radiative transfer model (e.g., Scoville \& Solomon 1974; Goldreich \& Kwan 1974) to constrain the excitation conditions. We adopt an escape probability of $\beta=\left(1-\mathrm{e}^{-\tau}\right) / \tau$, which implies a spherical geometry

\footnotetext{
4 The critical densities are calculated with $n_{\text {crit }}=A_{\mathrm{ul}} / \Sigma\left(C_{\mathrm{u} \neq 1}\right)$ as a function of kinetic temperature $T_{\text {kin }}$, under an optically thin assumption (Yang et al. 2010). Here $A$ is the Einstein coefficient for spontaneous emission, and $C$ is the collisional coefficient. Here we adopt $T_{\text {kin }}=20 \mathrm{~K}$. All state-to-state cross sections and rate coefficients for quenching are available in the LAMDA web site (http://home. strw . leidenuniv.nl/ moldata/; Schöier et al. 2005).
}

and an isothermal environment. While multiple phases of physical conditions should exist in the molecular cloud complexes of Circinus, it is difficult to disentangle them (but see below). We thus adopt homogeneous clouds in the LVG modeling to constrain the average physical properties of molecular gas.

We proceed with a three-dimensional parameter grid with regularly spaced kinetic temperature $\left(T_{\text {kin }}\right), \mathrm{H}_{2}$ number density $\left(n_{\mathrm{H}_{2}}\right)$, and fractional abundance versus velocity gradient $\left(x_{\mathrm{CO}} /(\mathrm{d} v / \mathrm{d} r)\right)$ as input, where $x_{\mathrm{CO}}$ is the abundance ratio of $\mathrm{CO}$ relative to $\mathrm{H}_{2}$. In the following analysis, $x_{{ }^{12}} \mathrm{CO}$ is fixed to $8 \times 10^{-5}$ (Frerking et al. 1982). The input parameter grid consists of $T_{\text {kin }}$ from $10^{1}$ to $10^{3} \mathrm{~K}, n_{\mathrm{H}_{2}}$ from $10^{2}$ to $10^{7} \mathrm{~cm}^{-3}$, and $\mathrm{d} v / \mathrm{d} r$ from $10^{0}$ to $10^{3} \mathrm{~km} \mathrm{~s}^{-1} \mathrm{pc}^{-1}$. We sample all these parameters with logarithmic steps of 0.1 . We adopt RADEX (van der Tak et al. 2007) to generate the model grids. We excluded all solutions with $\tau>100$ and the solutions did not reach convergence.

We adopt the ${ }^{12} \mathrm{CO}$ to ${ }^{13} \mathrm{CO}$ abundance ratio $\left(R_{1213}\right)$ to be 40 in the following analysis. It is intermediate between the values measured near the Galactic center and the solar circle (e.g., Wilson \& Matteucci 1992). This value is also consistent with the ratios derived in the active nuclear regions of nearby galaxies (e.g., NGC 1068, NGC 253, NGC 4945; Henkel \& Mauersberger 1993; Langer \& Penzias 1993; Wilson \& Rood 1994; Henkel et al. 2014). We do not adopt $R_{1213}=60-80$ given in Curran et al. (2001), because we obtained 25\% higher ${ }^{12} \mathrm{CO} J=3 \rightarrow 2$ flux (confirmed with several redundant observations) than their results. If the new ${ }^{12} \mathrm{CO} J=3 \rightarrow 2$ measurement is adopted in their model, higher excitation conditions will be obtained, and less $R_{1213}$ would be expected. We also tried $R_{1213}=80$ and 20 , which do not significantly change the final conclusions (see Table 7 and Sect. 4.7). The CO collisional rates are from Flower (2001), with an ortho/para $\mathrm{H}_{2}$ ratio of three. The output model grid includes excitation temperature, line brightness temperature, column density, and optical depth.

For each individual model, a $\chi^{2}$ value is calculated using differences in the ratios of line brightness temperatures obtained from the models and the observations. We derive $\chi^{2}$ with $\chi^{2}=\Sigma_{i}\left(1 / \sigma_{i}\right)^{2}\left(R_{\mathrm{obs}(i)}-R_{\operatorname{model}(i)}\right)^{2}$, where $R_{\mathrm{obs}}$ is the ratio of the measured line brightness temperatures, $\sigma_{\mathrm{i}}$ the error of the measured line ratio, and $R_{\text {model }}$ the ratio of the line brightness temperatures calculated by the LVG model.

\subsection{Single-component LVG modeling}

The comparatively small beam sizes of our new APEX data help us to probe the molecular gas properties in the innermost part of the galaxy. The beam size of the ${ }^{12} \mathrm{CO} J=3 \rightarrow 2$ data is $18^{\prime \prime}$, which is smaller than the diameter $\left(\sim 30-40^{\prime \prime}\right)$ of the S-F ring in the HST H $\alpha$ image (see Fig. 1). Therefore, as the first step, we analyze data exclusively taken with APEX to study the average physical conditions in the nuclear region. Because the published ${ }^{12} \mathrm{COJ}=1 \rightarrow 0$ and ${ }^{12} \mathrm{CO} J=2 \rightarrow 1$ data were measured with larger beams of $45^{\prime \prime}, 38^{\prime \prime}$, and $22^{\prime \prime}$ (see Table 6), we only model CO emissions with $J \geq 3$.

In our modeled grids, not all solutions have physical meaning. Therefore we set some priors to exclude solutions when they are either unphysical or contradictory to known information.

\subsubsection{Parameter restrictions}

We assume flat priors $(P)$ for $n_{\mathrm{H}_{2}}, \mathrm{~d} v / \mathrm{d} r$, and $T_{\text {kin }}$ with $P=1$ inside the ranges given in Sect. 4.1 and Table 5, and assign $P=0$ for solutions that do not match these prior criteria. In 
Table 5. Parameter restrictions for the LVG modeling.

$$
\begin{aligned}
& \text { 1) } T_{\mathrm{kin}}=10-1000 \mathrm{~K} \\
& \text { 2) } n\left(\mathrm{H}_{2}\right)=10^{2}-10^{7} \mathrm{~cm}^{-3} \\
& \text { 3) }(\mathrm{d} v / \mathrm{d} r)=1-360 \mathrm{~km} \mathrm{~s}^{-1} \mathrm{pc}^{-1}, x_{\mathrm{CO}}=8 \times 10^{-5} \\
& \text { 4) } K_{\mathrm{vir}}>1 \\
& \text { 5) } M_{\mathrm{H}_{2}} \leq 1.4 \times 10^{9} M_{\odot} \\
& \text { 6) For Low-J CO lines: } F_{18^{\prime \prime}} \leq F_{45^{\prime \prime}}{ }^{a} \\
& \text { 7) } \phi_{\mathrm{A}}<1^{b}
\end{aligned}
$$

Notes. ${ }^{(a)} F_{18^{\prime \prime}}$ and $F_{45^{\prime \prime}}$ denote modeled fluxes in an $18^{\prime \prime}$ beam and measured fluxes in a $45^{\prime \prime}$ beam, respectively. ${ }^{(b)} \phi_{\mathrm{A}}$ is the area filling factor.

the modeling of the parameter $x_{\mathrm{CO}} /(\mathrm{d} v / \mathrm{d} r)$, we keep $x_{\mathrm{CO}}$ constant (Sect. 4.1) and adjust the velocity gradient. Because of the degeneracy between the velocity gradient and the molecular abundance, modifications of $\mathrm{d} v / \mathrm{d} r$ have the same effect as changing $x_{\mathrm{CO}}$ for a given fixed $\mathrm{d} v / \mathrm{d} r$, which reflects the kinetic information of the modeled molecular gas. Varying $\mathrm{d} v / \mathrm{d} r$ helps us to find the thickness of the gas layer coupling ( $\left.r_{\text {coupling }}\right)$ in the radiative transfer via $r_{\text {coupling }}=\mathrm{d} v_{\text {cell }} /(\mathrm{d} v / \mathrm{d} r)$, where $\mathrm{d} v_{\text {cell }}=\left[\left(\mathrm{d} v_{\text {thermal }}\right)^{2}+\left(\mathrm{d} v_{\text {microturb }}\right)^{2}\right]^{1 / 2}$ is the intrinsic local line width of the gas cell where radiative coupling occurs (e.g., White 1977). We list the prior restrictions in Table 5 and discuss them below.

\section{Dynamical restriction $-K_{\mathrm{vir}}$, lower bound}

For a molecular cloud in virial equilibrium, random motions inside the cloud are compensated by self-gravity. If these motions are below a certain level, collapse should set in, however, even in the case of free-fall motions, velocities should not strongly deviate from those in a bound but non-collapsing system (e.g., Bertoldi \& McKee 1992; Krumholz \& McKee 2005). In the opposite case, however, when the gas experiences violent motion, as it may be in the case of shocks and outflows, the cloud could reach a highly supervirial state. The ratio between the modeled velocity gradient and that in the virialized state $\left(K_{\mathrm{vir}}\right.$; Appendix A) reflects the gas motion against self-gravity. The virialized state $K_{\text {vir }}$ is near unity in individual "normal" molecular clouds (e.g., Papadopoulos \& Seaquist 1999).

Subvirialization (i.e., $K_{\text {vir }} \ll 1$ ) is unphysical because gas motions inside GMCs can never be slower than what the cloud self-gravity dictates. The linear scales addressed here (several $100 \mathrm{pc}$ ) are dynamically dominated by galactic rotation, so that subvirialization can be firmly excluded. We therefore constrain $K_{\text {vir }} \geq 1$ throughout this paper.

\section{Dynamical restriction - $\mathrm{d} v / \mathrm{d} r$, upper bound}

The $\mathrm{H}_{2} \mathrm{O}$ masers measured with the Australia Telescope Long Baseline Array (Greenhill et al. 2003) indicate a particularly large velocity gradient, defined by the rotation of the maser disk around the central SMBH of Circinus. The velocity of the $\mathrm{H}_{2} \mathrm{O}$ masers varies by $\sim 400 \mathrm{~km} \mathrm{~s}^{-1}$ over a small warped disk of a diameter $\sim 80$ mas, which corresponds to $\sim 1.6$ pc. We derive an effective velocity gradient of $\mathrm{d} v / \mathrm{d} r_{\text {eff }}=$ $400 / 1.6=250 \mathrm{~km} \mathrm{~s}^{-1} \mathrm{pc}^{-1}$. This yields a convenient upper limit of $360 \mathrm{~km} \mathrm{~s}^{-1} \mathrm{pc}^{-1}$ to the velocity gradient in the LVG modeling, assuming that the adopted fractional $\mathrm{CO}$ abundance is correct within $\sim 50 \%$.

\section{Dynamical restriction - $M_{\mathrm{dyn}}$, upper bound}

We also discard solutions that have a total gas mass $\left(M_{\mathrm{gas}}\right)$ higher than the dynamical mass $\left(M_{\text {dyn }}\right)$. In Sect. 3.2.2, we have derived the dynamical mass within a galactocentric radius of $180 \mathrm{pc}$ to be $1.4 \pm 0.1 \times 10^{9} M_{\odot}$, which is the upper limit of

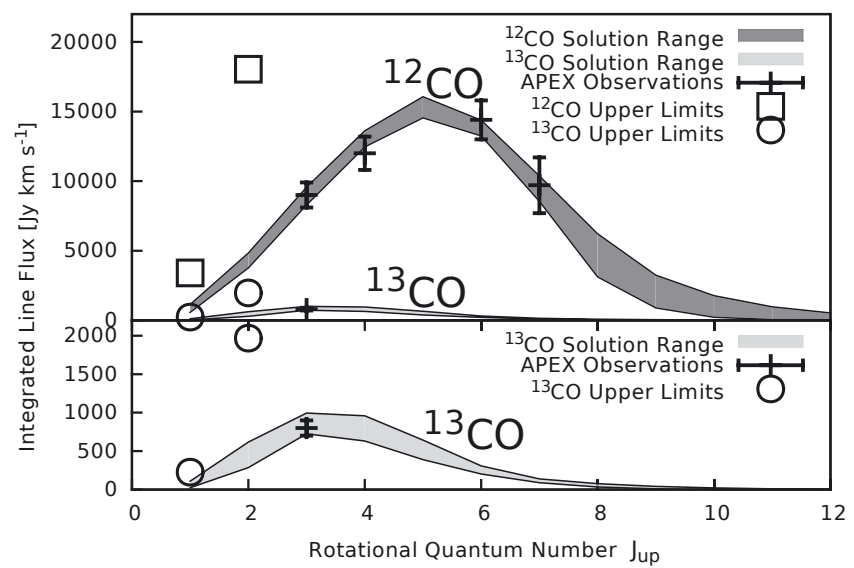

Fig. 7. Top: integrated flux densities of ${ }^{12} \mathrm{CO}$ and ${ }^{13} \mathrm{CO}$ transitions in the central region $\left(18^{\prime \prime}\right.$ in diameter) of Circinus. We plot the solution range of ${ }^{12} \mathrm{CO}$ (dark gray) and ${ }^{13} \mathrm{CO}$ (light gray) derived from singlecomponent LVG modeling of $\mathrm{CO}$ and ${ }^{13} \mathrm{CO}$ lines with $J \geq 3$. The range is selected from all solutions satisfying $\chi_{\text {red }}^{2}<1.5$ (or Likelihood $L>$ 0.6). We plot ${ }^{12} \mathrm{CO} J=2 \rightarrow 1,{ }^{12} \mathrm{CO} J=1 \rightarrow 0$, and their ${ }^{13} \mathrm{CO}$ isotopic transitions for beam sizes $>18^{\prime \prime}$ as upper limits (black boxes and circles) to our models. Bottom: integrated flux densities of ${ }^{13} \mathrm{CO}$, in a zoomed in view.

the interstellar gas mass. This mass limit corresponds to a limit of beam average $\mathrm{H}_{2}$ column density of $9 \times 10^{23} \mathrm{~cm}^{-2}$, for a $\mathrm{CO}$ abundance of $x_{\mathrm{CO}}=8 \times 10^{-5}$.

\section{Flux density limits - low-J CO}

The single LVG component models are based on the $\mathrm{CO}$ emission from the central $18^{\prime \prime}$ of Circinus, while the published ${ }^{12} \mathrm{CO} J=1 \rightarrow 0$ and ${ }^{12} \mathrm{CO} J=2 \rightarrow 1$ data were measured with larger beams of $45^{\prime \prime}, 38^{\prime \prime}$, and $22^{\prime \prime}$ (see Table 6 ). We set the constraint that the modeled flux densities of the ${ }^{12} \mathrm{CO} \mathrm{J}=1 \rightarrow 0$, $2 \rightarrow 1$ and their isotopic ${ }^{13} \mathrm{CO}$ lines cannot exceed the values observed with beam sizes $>18^{\prime \prime}$.

\subsubsection{The $\mathrm{CO}$ ladder in the central $18^{\prime \prime}$}

In Fig. 7 we show the observed CO spectral line energy distribution (SLED; velocity integrated flux density versus rotational transition number $J$ ) and our modeled SLED for the central $18^{\prime \prime}$ region. We also plot the line fluxes of the lower $\mathrm{CO}$ transitions $\left({ }^{12} \mathrm{CO} J=1 \rightarrow 0\right.$ and $\left.2 \rightarrow 1\right)$ and their ${ }^{13} \mathrm{C}$ isotopic lines as upper limits. For all successful models, the ${ }^{12} \mathrm{CO}$ SLEDs peak at ${ }^{12} \mathrm{CO} J=5 \rightarrow 4$, which cannot be observed with ground-based telescopes because of the very low atmospheric transmission at this frequency.

With five observational points and three fitting parameters, our modeling has two degrees of freedom (d.o.f.), so we discuss the general properties of the set of solutions satisfying $\chi_{\text {red }}^{2}=$ $\chi^{2} /($ d.o.f. -1$) \leq 1.5$, where $\chi_{\text {red }}^{2}$ is the reduced $\chi^{2}$. This corresponds to a likelihood limit of $L>0.6$. $L$ is defined by

$L_{\mathrm{i}}=\exp \left(-\chi_{\mathrm{i}}^{2} / 2\right) / L_{\max }$,

where $L_{\max }$ is the maximum likelihood for all solutions, which corresponds to the solution with the smallest value of $\chi^{2}$. The best fitting result has a $\chi_{\text {red }}^{2}$ of 0.5 , indicating that our adopted calibration error may be a bit conservative or that the number of degrees of freedom is not large enough to reach a lower limit of exactly unity (see Andrae et al. 2010).

The best fitting result (Table 7) indicates average physical conditions of $n_{\mathrm{H}_{2}} \sim 10^{3.2} \mathrm{~cm}^{-3}, T_{\text {kin }} \sim 200 \mathrm{~K}$, and 
Table 6. CO detections toward the central position of Circinus in the literature

\begin{tabular}{|c|c|c|c|c|c|}
\hline Transition & Telescope & $\begin{array}{l}\text { Resolution } \\
\left({ }^{\prime \prime}\right)\end{array}$ & $\begin{array}{c}W_{\mathrm{mb}} \\
\mathrm{Kkm} \mathrm{s}^{-1} \\
\end{array}$ & $\begin{array}{c}\text { Flux } \\
\text { Jy km s}^{-1}\end{array}$ & $\eta_{\mathrm{mb}}$ \\
\hline${ }^{12} \mathrm{CO} J=1 \rightarrow 0^{a}$ & SEST & $43^{\prime \prime}$ & $128 \pm 1.2$ & 2650 & 0.77 \\
\hline${ }^{12} \mathrm{COJ}=1 \rightarrow 0^{b}$ & SEST & & $185 \pm 15$ & 3350 & 0.67 \\
\hline${ }^{12} \mathrm{COJ}=1 \rightarrow 0^{c}$ & MOPRA & & 14 & - & - \\
\hline${ }^{12} \mathrm{CO} J=1 \rightarrow 0^{d}$ & SEST & & 156 & 3050 & 0.72 \\
\hline${ }^{12} \mathrm{COJ}=1 \rightarrow 0^{e}$ & SEST & & $180 \pm 10$ & 3500 & - \\
\hline${ }^{12} \mathrm{CO} J=1 \rightarrow 0^{f}$ & SEST & & 0 & 850 & 0.7 \\
\hline Adopted & - & $45^{\prime \prime}$ & $180 \pm 10$ & 3500 & - \\
\hline${ }^{13} \mathrm{CO} J=1 \rightarrow 0^{a}$ & SEST & $43^{\prime \prime}$ & $11.2 \pm 0.2$ & 230 & 0.77 \\
\hline${ }^{13} \mathrm{COJ}=1 \rightarrow 0^{b}$ & SEST & & & 210 & 0.68 \\
\hline${ }^{13} \mathrm{CO} J=1 \rightarrow 0^{d}$ & SEST & & 13 & 250 & 0.72 \\
\hline${ }^{13} \mathrm{CO} J=1 \rightarrow 0^{e}$ & SEST & & $12 \pm 1$ & 230 & - \\
\hline Adopted & SEST & & 11.2 & 230 & - \\
\hline${ }^{12} \mathrm{CO} J=2 \rightarrow 1^{d}$ & SEST & $45^{\prime \prime}$ & & 12300 & 0.6 \\
\hline${ }^{12} \mathrm{COJ}=2 \rightarrow 1^{e}$ & SEST & & $220 \pm$ & 5400 & 0.6 \\
\hline${ }^{12} \mathrm{COJ}=2 \rightarrow 1^{g}$ & SEST & & 17 & 13000 & 0.6 \\
\hline Adopted & SEST & $45^{\prime \prime}$ & 144 & $14200^{i}$ & - \\
\hline${ }^{13} \mathrm{CO} J=2 \rightarrow 1^{d}$ & SEST & $45^{\prime \prime}$ & 12.5 & 1280 & 0.60 \\
\hline${ }^{13} \mathrm{CO} J=2 \rightarrow 1^{e}$ & SEST & & & 590 & 0.6 \\
\hline${ }^{13} \mathrm{CO} J=2 \rightarrow 1^{g}$ & SEST & & 1 & 1390 & 0.6 \\
\hline Adopted & SEST & $45^{\prime \prime}$ & 12.5 & 1300 & - \\
\hline${ }^{12} \mathrm{CO} J=3 \rightarrow 2^{d}$ & SEST & $45^{\prime \prime}$ & 70 & 20400 & 0.33 \\
\hline${ }^{12} \mathrm{COJ}=3 \rightarrow 2^{e}$ & SEST & 15 & $230 \pm$ & 7400 & 0.33 \\
\hline${ }^{12} \mathrm{CO} J=3 \rightarrow 2^{g}$ & APEX & 45 & $140 \pm$ & 25000 & 0.73 \\
\hline Adopted & APEX & $45^{\prime \prime}$ & $140 \pm 20$ & 25000 & - \\
\hline${ }^{12} \mathrm{CO} J=4 \rightarrow 3^{h}$ & NANTEN-2 & $38^{\prime \prime}$ & 58 & 12600 & 0.5 \\
\hline${ }^{12} \mathrm{CO} J=3 \rightarrow 2^{g}$ & APEX & $45^{\prime \prime}$ & $80 \pm$ & 25000 & 0.6 \\
\hline Adopted & APEX & $45^{\prime \prime}$ & $80 \pm 15$ & 25000 & - \\
\hline
\end{tabular}

Notes. ${ }^{(a)}$ Aalto et al. (1991); ${ }^{(b)}$ Israel et al. (1992); ${ }^{(c)}$ Elmouttie et al. (1997); ${ }^{(d)}$ Curran et al. (1998); ${ }^{(e)}$ Curran et al. (2001a); ${ }^{(f)}$ Curran et al. (2001b); ${ }^{(g)}$ this work; ${ }^{(h)}$ Hitschfeld et al. (2007); ${ }^{(i)}$ We adopt the CO $J=2 \rightarrow 1$ to $1 \rightarrow 0$ line ratio in the $45^{\prime \prime}$ region, and the integrated intensity of ${ }^{12} \mathrm{CO} J=1 \rightarrow 0$ in Curran et al. (2008). The line fluxes are derived from $T_{\mathrm{A}}^{\star}$ by adopting the telescope efficiencies of the SEST with $27 \mathrm{Jy} / \mathrm{K}$, $41 \mathrm{Jy} / \mathrm{K}$, and $98 \mathrm{Jy} / \mathrm{K}$ at $115 \mathrm{GHz}, 230 \mathrm{GHz}$, and $346 \mathrm{GHz}$, respectively. For the NANTEN-2 $4 \mathrm{~m}$ telescope, a Jy/K factor for $T_{\mathrm{mb}}$ of $216 \mathrm{has}$ been assumed.

$\mathrm{d} v / \mathrm{d} r \sim 3 \mathrm{~km} \mathrm{~s}^{-1} \mathrm{pc}^{-1}$. Various sets of degenerated parameter combinations satisfy $\chi_{\text {red }}^{2}<1.5$, and these solutions also provide reasonable fittings. The degeneracy not only affects $T_{\text {kin }}$ and $n_{\mathrm{H}_{2}}$, but also $\mathrm{d} v / \mathrm{d} r$. For example, the "dense solution" has $n_{\mathrm{H}_{2}}=10^{3.7} \mathrm{~cm}^{-3}, T_{\text {kin }}=125 \mathrm{~K}$, and $\mathrm{d} v / \mathrm{d} r=$ $20.0 \mathrm{~km} \mathrm{~s}^{-1} \mathrm{pc}^{-1}$, with a $\chi_{\text {red }}^{2} \sim 1.5$. A "hot solution" with $n_{\mathrm{H}_{2}}=$ $10^{3.2} \mathrm{~cm}^{-3}, T_{\text {kin }}=250 \mathrm{~K}$, and $\mathrm{d} v / \mathrm{d} r=5.0 \mathrm{~km} \mathrm{~s}^{-1} \mathrm{pc}^{-1}$ achieves a similar $\chi^{2}$ value. In summary, these solutions encompass a range of $10^{2.7} \mathrm{~cm}^{-3}<n_{\mathrm{H}_{2}}<10^{3.8} \mathrm{~cm}^{-3}, 80 \mathrm{~K}<T_{\text {kin }}<400 \mathrm{~K}$, $1 \mathrm{~km} \mathrm{~s}^{-1} \mathrm{pc}^{-1}<\mathrm{d} v / \mathrm{d} r<25 \mathrm{~km} \mathrm{~s}^{-1} \mathrm{pc}^{-1}$.

We calculate the area filling factors $\left(\phi_{\mathrm{A}}\right)$ with the ratio of the observed and the modeled line intensities, by $\phi_{\mathrm{A}}=$ $\Sigma T_{\text {obs }}(i) / \Sigma T_{\mathrm{LVG}}(i)$, where $i$ is the upper level of the transitions. We find a narrow range of $\phi_{\mathrm{A}}$ between $1.8 \%$ and $2.3 \%$. We calculate the equivalent radius with: $r_{\mathrm{eff}}=\sqrt{\phi_{\mathrm{A}}} r_{\text {beam }} / 2$, where $\phi_{\mathrm{A}}$ is the beam filling factor, and $r_{\text {beam }}$ is the physical size covered by the telescope beam. The corresponding effective emission sizes are between $10 \mathrm{pc}$ and $15 \mathrm{pc}$ in diameter. A detailed likelihood analysis is presented in Appendix B.

\subsection{Two-component modeling in the central $45^{\prime \prime}$ region}

In this section, we explore the physical conditions in the central $45^{\prime \prime}(900 \mathrm{pc})$ region with LVG modeling. We combine our mid- $J$ CO measurements with the low- $J$ CO data from the literature to perform a global fitting. We convolve all $\mathrm{CO}$ maps to
Table 7. Physical parameters of single-component fitting in the central $18^{\prime \prime}$ region, with $\chi_{\text {red }}^{2}<1.5$.

\begin{tabular}{llcc}
\hline \hline Parameters & Min & Max & Best fitting \\
\hline$\chi_{\text {red }}^{2}$ & 0.5 & 1.5 & 0.5 \\
$\log \left(\right.$ Density) $\left[\mathrm{cm}^{-3}\right]$ & 2.7 & 3.8 & 3.2 \\
Temperature $[\mathrm{K}]$ & 80 & 400 & 200 \\
$\mathrm{~d} v / \mathrm{d} r\left[\mathrm{~km} \mathrm{~s}^{-1} \mathrm{pc}^{-1}\right]$ & 1.0 & 25 & 3.0 \\
$N(\mathrm{CO})_{\text {model }^{a}}\left[10^{19} \mathrm{~cm}^{-2}\right]$ & 3.1 & 7.8 & 4.9 \\
$N(\mathrm{CO})_{\text {beam }}{ }^{b}\left[10^{17} \mathrm{~cm}^{-2}\right]$ & 5.7 & 17 & 9.2 \\
$N\left(\mathrm{H}_{2}\right)_{\text {beam }}{ }^{c}\left[10^{21} \mathrm{~cm}^{-2}\right]$ & 7.1 & 21 & 11.5 \\
$M_{\mathrm{H}_{2}}{ }^{d}\left[10^{7} M_{\odot}\right]$ & 0.7 & 2.2 & 1.3 \\
Area Filling Factor $\left(\phi_{\mathrm{A}}\right)$ & $1.8 \%$ & $2.3 \%$ & $1.9 \%$ \\
\hline
\end{tabular}

Notes. (a) $N(\mathrm{CO})_{\text {model }}$ is the $\mathrm{CO}$ column density derived in the LVG models. ${ }^{(b)} N(\mathrm{CO})_{\text {beam }}$ is the $\mathrm{CO}$ column density diluted by the area filling factor $\left(\phi_{\mathrm{A}}\right){ }^{\left({ }^{c}\right)} N\left(\mathrm{H}_{2}\right)_{\text {beam }}$ is the beam average $\mathrm{H}_{2}$ column density. (d) $M\left(\mathrm{H}_{2}\right)$ is the molecular gas mass within a beam size of $18^{\prime \prime}$, using $M_{\mathrm{H}_{2}}=1.36 \times \frac{N(\mathrm{CO})_{\text {beam }}}{x_{\mathrm{CO}}} \times\left(\pi r^{2}\right)$, where $r$ is the radius of the beam, $180 \mathrm{pc}$, and $x_{\mathrm{CO}}$ is the $\mathrm{CO}$ to $\mathrm{H}_{2}$ abundance, $8 \times 10^{-5}$ (Frerking et al. 1982). The helium mass is included in $M\left(\mathrm{H}_{2}\right)$. We also examined the molecular gas mass using a $R_{1213}$ of 89 , which gives a range from $0.7 \times 10^{7} M_{\odot}$ to $2.6 \times 10^{7} M_{\odot}$, and the best-fit molecular gas mass is $1.7 \times 10^{7} M_{\odot}$.

the beam size of the SEST at the frequency of ${ }^{12} \mathrm{CO} J=1 \rightarrow 0$, i.e., $F W H M=45^{\prime \prime}$. The intensity and resolution of these lines 
are tabulated in Table 6 . We convolve the ${ }^{13} \mathrm{CO} J=3 \rightarrow 2$ emission to a resolution of $45^{\prime \prime}$, assuming that the distribution of ${ }^{13} \mathrm{CO} J=3 \rightarrow 2$ is the same as that of ${ }^{12} \mathrm{CO} J=3 \rightarrow 2$.

We tried to fit $\mathrm{CO}$ ladders in the central $45^{\prime \prime}$ with a single LVG component first, however, it does not produce a good fit. This is not surprising since the modeling results in previous studies (e.g., Kamenetzky et al. 2012; Hailey-Dunsheath et al. 2012; Rigopoulou et al. 2013) have shown that the coexistence of multiple excitation gas components in nearby galaxies (also see $\mathrm{Lu}$ et al. 2014). Therefore, we use two LVG components to model the gas excitation in the central $45^{\prime \prime}$ region.

In the two-component LVG modeling, we assume that both components have the same chemical abundance: $x_{\mathrm{CO}}=8 \times$ $10^{-5}$, and $R_{1213}=40$. Each component has its own $n_{\mathrm{H}_{2}}, T_{\text {kin }}$, $\mathrm{d} v / \mathrm{d} r$, and a relative contribution to the measured line intensities. We list the priors in the two-component models in Table 8.

We analyze with the same grids as in the single-component fitting (see Sect. 4.2) and model the line intensities for both components simultaneously. We assume that the two-components have independent excitation conditions. The sum of the twocomponents should match the observed SLED. To construct the contributions of the two-components, we assume that the LE and the higher-excitation (HE) components are diluted by the filling factors of $\phi_{\mathrm{LE}}$ and $\phi_{\mathrm{HE}}$, respectively. The observed main beam temperature can be modeled with: $T_{\mathrm{obs}}=T_{\mathrm{LE}} \times$ $\phi_{\mathrm{LE}}+T_{\mathrm{HE}} \times \phi_{\mathrm{HE}}=C \times\left[(1-R) \times T_{\mathrm{LE}}+R \times T_{\mathrm{HE}}\right]$, where $C\left(=\phi_{\mathrm{LE}}+\phi_{\mathrm{HE}}\right)$ is a constant number for each model, and $T_{\mathrm{LE}}$ and $T_{\mathrm{HE}}$ are the modeled line intensities for the low- and high-excitation components, respectively. The relative ratio $R$ ( $=\frac{\phi_{\mathrm{HE}}}{\phi_{\mathrm{HE}}+\phi_{\mathrm{LE}}}$, and $\left.0<R<1\right)$ reflects the contribution relative to the total line intensity, and $R$ is calculated from $5 \%$ to $95 \%$ with a step size of $5 \%$. The relative mass contributions of these phases can be expressed by: $M_{\mathrm{H}_{2}(\mathrm{LE})} / M_{\mathrm{H}_{2}(\mathrm{HE})}=\phi_{\mathrm{LE}} / \phi_{\mathrm{HE}} \times\left(T_{\mathrm{LE}} / T_{\mathrm{TE}}\right) \times$ $\left[X_{\mathrm{CO}(\mathrm{LE})} / X_{\mathrm{CO}(\mathrm{HE})}\right]$, where $X_{\mathrm{CO}(\mathrm{LE}, \mathrm{HE})}$ are the $X_{\mathrm{CO}}$ factors for those phases (Papadopoulos et al. 2012a):

$X_{\mathrm{CO}}=\frac{3.25}{\sqrt{\alpha}} \frac{n\left(\mathrm{H}_{2}\right)}{T_{\mathrm{b}}(J=1 \rightarrow 0)} K_{\mathrm{vir}}^{-1}\left(\frac{M_{\odot}}{\mathrm{Kkm} \mathrm{s}^{-1} \mathrm{pc}^{2}}\right)$,

where $\alpha=0.55-2.4$, depending on the assumed cloud density profile, $T_{\mathrm{b}}(J=1 \rightarrow 0)$ is the brightness temperature of ${ }^{12} \mathrm{CO} J=$ $1 \rightarrow 0$.

With nine measurements and seven fitting parameters, we discuss the general properties of the set of solutions satisfying likelihood $L \geq 0.7$. In Fig. 8 we plot the line flux ranges of all the accepted solutions. For all good solutions, the ${ }^{12} \mathrm{CO} J=1 \rightarrow 0$ and $2 \rightarrow 1$ intensities of the LE component are much higher than those of the HE component. The ${ }^{12} \mathrm{COJ}=3 \rightarrow 2$ and $4 \rightarrow 3$ intensities profit by similar contributions from both components. The ${ }^{12} \mathrm{CO} J=6 \rightarrow 5$ and $7 \rightarrow 6$ emission are dominated by the HE component.

We find that in the solutions with the lowest $\chi^{2}$, the relative contribution ratio is $R \sim 0.15$. Setting this ratio as the basis for the two-components, we probe the ranges of physical parameters in the following analysis. The best-fit model shows a HE component of $T_{\text {kin }} \sim 60 \mathrm{~K}, n_{\mathrm{H}_{2}} \sim 10^{4.2} \mathrm{~cm}^{-3}$, and $\mathrm{d} v / \mathrm{d} r \sim$ $50 \mathrm{~km} \mathrm{~s}^{-1} \mathrm{pc}^{-1}$, and a LE component of $T_{\text {kin }} \sim 30 \mathrm{~K}, n_{\mathrm{H}_{2}} \sim 10^{3.0}$ and $\mathrm{d} v / \mathrm{d} r \sim 6 \mathrm{~km} \mathrm{~s}^{-1} \mathrm{pc}^{-1}$ (for details, see Appendix C). The best fit shows an equivalent emission radius of $\sim 20 \mathrm{pc}$ for the HE component, which is larger than the effective emission radius of $10-15 \mathrm{pc}$ found in the previous single-component fitting.

The best solutions show that the HE component model has a velocity gradient about 10 times higher than the LE component. This indicates that more violent kinematics are associated

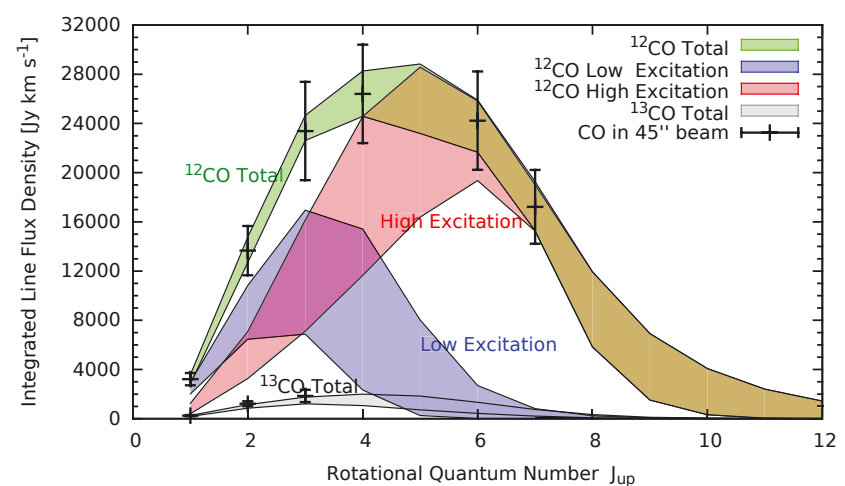

Fig. 8. Integrated flux densities of ${ }^{12} \mathrm{CO}$ and ${ }^{13} \mathrm{CO}$ in the central $45^{\prime \prime}$ region of the Circinus galaxy. The shadowed regions are the ranges of the best fitting results derived from the two-component LVG modeling. The high- and low-excitation components and the total integrated ${ }^{12} \mathrm{CO}$ flux densities are plotted in red, blue, and green. The total integrated ${ }^{13} \mathrm{CO}$ flux densities are plotted in gray.

Table 8. Parameter restrictions for the two-component LVG modeling.

\begin{tabular}{l}
\hline 1) $T_{\mathrm{kin}}=10-1000 \mathrm{~K}$ \\
2) $n\left(\mathrm{H}_{2}\right)=10^{2}-10^{7} \mathrm{~cm}^{-3}$ \\
3) $1 \leq \mathrm{d} v / \mathrm{d} r \leq 360 \mathrm{~km} \mathrm{~s}^{-1} \mathrm{pc}^{-1 a}, x_{\mathrm{CO}}=8 \times 10^{-5}$ \\
4) $K_{\mathrm{vir}}>1$ \\
5) $M_{\mathrm{H} 2}<M_{\mathrm{dyn}}=3.6 \times 10^{9} M_{\odot}^{b}$ \\
6) $\phi_{\mathrm{A}}<1^{c}$
\end{tabular}

Notes. ${ }^{(a)} \mathrm{d} v / \mathrm{d} r$ limit from Sect. 3.2.2. ${ }^{(b)} M_{\text {dyn }}$ is the dynamical mass, $M_{\text {dyn }}=3.6 \times 10^{9} M_{\odot}$ for a beam size of $45^{\prime \prime}$ (see Sect. 3.2.2). ${ }^{(c)} \phi_{\mathrm{A}}$ are area filling factors for both components derived by LVG modeling.

with the HE component, and that the molecular gas in the inner $18^{\prime \prime}$ region (Sect. 4.2.2) is in a state of high excitation because a high $\mathrm{d} v / \mathrm{d} r$ is expected in the center (e.g., Tan et al. 2011). We summarize the results of the two-component fittings in Table 9. Although we obtain a lower temperature, the best density solution of the LE component is also similar to the fitting results of the low- $J$ transitions of $\mathrm{CO}$ in Curran et al. (2001), where they find $\mathrm{T}_{\text {kin }}=50-80 \mathrm{~K}, n_{\mathrm{H} 2}=2 \times 10^{3} \mathrm{~cm}^{-2}$, and $\mathrm{d} v / \mathrm{d} r=$ $10 \mathrm{~km} \mathrm{~s}^{-1} \mathrm{pc}^{-1}$.

\subsection{Does the HE component arise from the $18^{\prime \prime}$ region?}

Single LVG component fitting of the inner $18^{\prime \prime}$ region leads to an order of magnitude lower density and four times lower temperature than the corresponding parameters derived from the HE component in the $45^{\prime \prime}$ region. If the inner $18^{\prime \prime}$ dominates the HE component, why are there such large discrepancies? Does the HE component mainly arise from the $18^{\prime \prime}$ region? First, the HE component in the $45^{\prime \prime}$ region cannot entirely arise from the $18^{\prime \prime}$ nuclear region because the ring contributes about $35 \%$ and $45 \%$ fluxes of ${ }^{12} \mathrm{CO} J=6 \rightarrow 5$ and $7 \rightarrow 6$, respectively (see Sect. 4.5). Second, the single LVG component modeling only reflects the average physical conditions in this region, where the gas may not be dominated by the HE component. In the $18^{\prime \prime}$ region the mid- $J$ transitions (especially ${ }^{12} \mathrm{CO} J=3 \rightarrow 2$ and $4 \rightarrow 3$ ) are also likely contaminated by the lower excitation component, which may provide a large amount of diffuse cold gas along the line of sight. Third, the degeneracies between temperature, density, and velocity gradient are responsible for the difference. A component with lower density and higher temperature 
Table 9. Physical parameters of two-component fitting in the central $45^{\prime \prime}$ region.

\begin{tabular}{lllllll}
\hline \hline Parameter & \multicolumn{2}{c}{ Low-excitation component } & \multicolumn{3}{c}{ High-excitation component } \\
\hline & $\min$ & $\max$ & best fitting & $\min$ & $\max$ & best fitting \\
\hline Density $\left[\mathrm{cm}^{-3}\right]$ & $10^{2.8}$ & $10^{3.5}$ & $10^{3.0}$ & $10^{3.3}$ & $10^{5.3}$ & $10^{4.2}$ \\
Temperature $[\mathrm{K}]$ & 20 & 80 & 30 & 40 & 400 & 60 \\
$\mathrm{~d} v / \mathrm{d} r\left[\mathrm{~km} \mathrm{~s}^{-1} \mathrm{pc}^{-1}\right]$ & 5 & 25 & 6 & 3 & 300 & 50 \\
$N(\mathrm{CO})_{\text {beam }}\left[10^{17} \mathrm{~cm}^{-2}\right]^{a}$ & 2.7 & 5.0 & 3.9 & 0.9 & 2.3 & 1.4 \\
$N(\mathrm{CO})_{\text {model }}\left[10^{19} \mathrm{~cm}^{-2}\right]^{b}$ & 0.9 & 1.5 & 1.2 & 1.5 & 4.6 & 2.3 \\
$M_{\mathrm{H}_{2}}\left[10^{7} M_{\odot}\right]^{c}$ & 4.3 & 8.6 & 6.6 & 1.2 & 4.0 & 2.3 \\
Filling Factor & $2.6 \%$ & $4.4 \%$ & $3.3 \%$ & $0.44 \%$ & $0.82 \%$ & $0.58 \%$ \\
\hline
\end{tabular}

Notes. ${ }^{(a)} N(\mathrm{CO})_{\text {beam }}$ is the $\mathrm{CO}$ column density diluted by the filling factor $\left(\phi_{\mathrm{A}}\right) .{ }^{(b)} N(\mathrm{CO})_{\text {model }}$ is the CO column density derived in the LVG models. ${ }^{(c)} M_{\mathrm{H}_{2}}$ is calculated with the beam size of $45^{\prime \prime}$, using $M_{\mathrm{H}_{2}}=1.36 \times \frac{N(\mathrm{CO})_{\text {beam }}}{x_{\mathrm{CO}}} \times\left(\pi r^{2}\right)$, where $r$ is the radius of the beam, $450 \mathrm{pc}$. $x_{\mathrm{CO}}$ is the CO to $\mathrm{H}_{2}$ abundance ratio, $8 \times 10^{-5}$ (Frerking et al. 1982). The helium mass is included in $M_{\mathrm{H}_{2}}$.

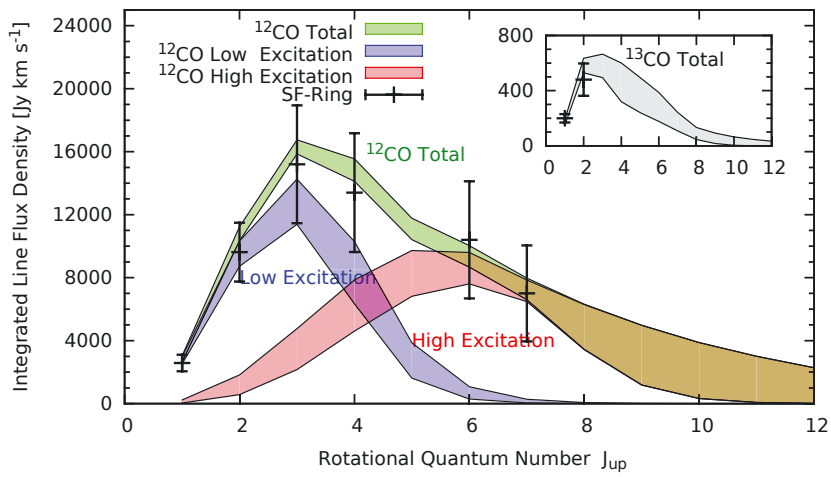

Fig. 9. Integrated flux densities of ${ }^{12} \mathrm{CO}$ and ${ }^{13} \mathrm{CO}$ in the S-F ring region of the Circinus galaxy. The shadowed regions are the ranges of the fittings satisfying likelihood $L>0.8$, derived from the two-component LVG modeling. The high- and low-excitation components and the total integrated ${ }^{12} \mathrm{CO}$ flux densities are plotted in red, blue, and green. The total ${ }^{13} \mathrm{CO}$ flux densities are plotted in gray.

can produce similar CO SLEDs to a component with higher density but lower temperature.

\subsection{Gas excitation in the S-F ring}

For the ${ }^{12} \mathrm{CO} J=6 \rightarrow 5$ and $J=7 \rightarrow 6$ transitions, we find that aboutwh $35 \%-45 \%$ of the CO fluxes come from the S-F ring region (diameter $\left.=18^{\prime \prime}-45^{\prime \prime}\right)$, and the rest comes from the center. This indicates that the HE component derived in the twocomponents decomposition is not likely from the nuclear region alone, and a large amount of highly excited gas seems to exist in the S-F ring.

Benefiting from the mapping observations, we derive $\mathrm{CO}$ emission in the S-F ring region by subtracting line fluxes, which were derived from the best model obtained in Sect. 4.2, in the central $18^{\prime \prime}$ region from those in the $45^{\prime \prime}$ region. We fit the CO residual with two LVG components, to model the gas excitation exclusively in the S-F ring region, as can be seen in Fig. 9. We fit the model with all transitions of ${ }^{12} \mathrm{CO}$ and ${ }^{13} \mathrm{COJ}=1 \rightarrow 0,2 \rightarrow 1$. Because ${ }^{13} \mathrm{COJ}=3 \rightarrow 2$ was not measured in the $\mathrm{S}-\mathrm{F}$ ring region, we cannot make any constraint on it. The $J=1 \rightarrow 0$ and $2 \rightarrow 1$ transitions are derived from the residual by subtracting the LVG model in the central $18^{\prime \prime}$ region from the fluxes in the $45^{\prime \prime}$ region. The best fit of the ring shows a HE component of $n_{\mathrm{H}_{2}} \sim 10^{4.1} \mathrm{~cm}^{-3}, T_{\text {kin }} \sim 125 \mathrm{~K}$, and an LE component of $n_{\mathrm{H}_{2}} \sim 10^{2.9} \mathrm{~cm}^{-3}, T_{\text {kin }} \sim 30 \mathrm{~K}$, as shown in Table 10 .

\subsection{Comparison with NGC 1068}

Circinus and NGC 1068 have many similarities. They are both nearby Seyfert galaxies, and contain gas-rich nuclei and molecular S-F rings. Although Circinus has a much smaller distance ( 4 Mpc) than NGC 1068 ( 14.4 Mpc Bland-Hawthorn et al. 1997), the angular sizes of the gas-rich region in these two galaxies are both about 40" (in diameter). Both of them have strong S-F activities in their centers, and are fed by large amounts of molecular material (e.g., Curran et al. 2001; Hailey-Dunsheath et al. 2012).

In NGC 1068, the inner ends of the S-F spiral arms lead to a large $\mathrm{S}-\mathrm{F}$ ring of diameter $\sim 2.3 \mathrm{kpc}$ (e.g., Schinnerer et al. 2000; Gallimore et al. 2001). Closer to the center there is a CND of diameter $\sim 300 \mathrm{pc}$, seen most prominently in line emission of dense gas tracers (e.g., Schinnerer et al. 2000; Krips et al. 2011). Between the S-F ring and the CND there is a gap region deficient in molecular gas (e.g., Helfer et al. 2003; Schinnerer et al. 2000; Tacconi et al. 1997; Tsai et al. 2012). Spinoglio et al. (2012) modeled the excitation conditions with the CO SLED deduced from the Herschel observations and found an LE component $\left(T_{\text {kin }}=120 \mathrm{~K}, n_{\mathrm{H}_{2}}=10^{2.8} \mathrm{~cm}^{-3}\right)$ associated with an extended source (the $\mathrm{S}-\mathrm{F}$ ring), a medium excitation (ME) component $\left(T_{\text {kin }}=100 \mathrm{~K}, n_{\mathrm{H}_{2}}=10^{4.6} \mathrm{~cm}^{-3}\right)$ associated with the CND, and a $\mathrm{HE}$ component $\left(T_{\text {kin }}=150 \mathrm{~K}, n_{\mathrm{H}_{2}}=10^{5.7} \mathrm{~cm}^{-3}\right)$ possibly arises from the central few pc heated by the AGN (e.g., Hailey-Dunsheath et al. 2012).

Considering the whole inner $45^{\prime \prime}$ region of Circinus, the LE component has $n_{\mathrm{H}_{2}} \sim 10^{3.0} \mathrm{~cm}^{-3}$, similar to the LE component derived from the extended emission in NGC 1068 and the central region of the Milky Way (e.g., Spinoglio et al. 2012; Ott et al. 2014). The temperature of the LE component, however, is $T_{\text {kin }} \sim 30 \mathrm{~K}$, which is much lower than the LE component in NGC 1068, indicating that Circinus may have lower excitation conditions.

On the other hand, the HE component in Circinus is also characterized by a similar density and a lower temperature compared to the ME component in NGC 1068, which is from the CND region, and is fitted using the high- $J$ transitions $(J=9 \rightarrow 8$ to $13 \rightarrow 12$ ) in a $17^{\prime \prime}$ region (i.e., Spinoglio et al. 2012). The velocity gradient of the HE component spans a large range and all solutions indicate that this gas component is in a highly supervirialized state.

In NGC 1068, the LVG modeling was made step by step from higher to lower excitation components. Each component was fitted individually, after subtracting the higher excitation components. In Circinus we fit the two-components 
Table 10. Physical parameters of two-component fitting of the S-F ring at diameter $18^{\prime \prime}<D<45^{\prime \prime}$, with Likelihood $L>0.8$ (see Eq. (1)).

\begin{tabular}{lllllll}
\hline \hline Parameter & \multicolumn{2}{c}{ Low-excitation component } & \multicolumn{3}{c}{ High-excitation component } \\
\hline & $\min$ & $\max$ & best fitting & $\min$ & $\max$ & best fitting \\
\hline${\text { Density }\left[\mathrm{cm}^{-3}\right]}_{\text {Temperature }[\mathrm{K}]}$ & $10^{2.9}$ & $10^{3.6}$ & $10^{3.2}$ & $10^{3.0}$ & $10^{5.0}$ & $10^{4.1}$ \\
$\mathrm{~d} v / \mathrm{d} r\left[\mathrm{~km} \mathrm{~s}^{-1} \mathrm{pc}^{-1}\right]$ & 15 & 40 & 20 & 15 & 300 & 60 \\
$N(\mathrm{CO})_{\text {beam }}\left[10^{17} \mathrm{~cm}^{-2}\right]^{a}$ & 6.0 & 40 & 10 & 8.0 & 160 & 40 \\
$N(\mathrm{CO})_{\text {model }}\left[10^{19} \mathrm{~cm}^{-2}\right]^{b}$ & 2.2 & 4.2 & 3.7 & 0.3 & 1.6 & 0.6 \\
$M_{\mathrm{H}_{2}}\left[10^{7} M_{\odot}{ }^{c}\right.$ & 3.8 & 1.5 & 1.2 & 1.5 & 4.6 & 2.3 \\
Filling Factor $\left(\phi_{\mathrm{A}}\right)$ & $2.4 \%$ & $4.6 \%$ & $3.4 \%$ & 0.6 & 2.3 & 1.0 \\
\hline
\end{tabular}

Notes. ${ }^{(a)} N(\mathrm{CO})_{\text {beam }}$ is the $\mathrm{CO}$ column density diluted by the filling factor $\left(\phi_{\mathrm{A}}\right) .{ }^{(b)} N(\mathrm{CO})_{\text {model }}$ is the CO column density derived in the LVG models. (c) $M_{\mathrm{H}_{2}}$ is calculated with $M_{\mathrm{H}_{2}}=1.36 \times \frac{N(\mathrm{CO})_{\text {beam }}}{x_{\mathrm{CO}}} \times\left(\pi r^{2}\right)$, where $r$ is the effective radius of the beam, $r=\sqrt{\left(450^{2}-180^{2}\right)}=412 \mathrm{pc} . x_{\mathrm{CO}}$ is the CO to $\mathrm{H}_{2}$ abundance, $8 \times 10^{-5}$ (Frerking et al. 1982). The helium mass is included in $M_{\mathrm{H}_{2}}$ by adopting a factor of 1.36.

simultaneously, which allows for a much larger parameter space. The different fitting methods could also introduce differences. High angular resolution observations of multiple- $J$ CO transitions in Circinus are needed to fully resolve the gas phase distribution and fully test the above scenarios.

\subsection{Molecular gas mass}

We calculate molecular gas mass from the LVG models derived from previous sections. RADEX gives the column density $N_{\mathrm{CO}}$ without beam dilution. We convert it to a beamaveraged ${ }^{12} \mathrm{CO}$ column density $\left\langle N_{\mathrm{CO}}\right\rangle$, which is then further converted to the column density of molecular hydrogen with the assumed $\mathrm{CO}$ abundance of $x_{\mathrm{CO}}=8 \times 10^{-5}$. The molecular gas mass is derived with

$M_{\mathrm{H}_{2}}=1.36 \times m_{\mathrm{H}_{2}} \frac{\left\langle N_{\mathrm{CO}}\right\rangle}{x_{\mathrm{CO}}} \frac{\int T_{\mathrm{mb}} \mathrm{d} v \times A}{W}$,

where $m_{\mathrm{H}_{2}}$ is the mass of a single $\mathrm{H}_{2}$ molecule and the factor of 1.36 accounts for the mass of helium in the molecular clouds. The beam area $A=\pi r_{0}^{2}$, where $r_{0}$ is radius of the beam. The velocity integrated line intensity $W$ is calculated in the LVG models. The main beam temperature $T_{\mathrm{mb}}$ is obtained from the observations (Ward et al. 2003).

In the single-component modeling, the beam size corresponds to a region with radius $r_{0}$ of (central) $180 \mathrm{pc}$. From the best fitting results (see Sect. 4.2), a velocity gradient $\mathrm{d} v / \mathrm{d} r \sim$ $3 \mathrm{~km} \mathrm{~s}^{-1} \mathrm{pc}^{-1}$, and a density $n_{\mathrm{H}_{2}} \sim 10^{3.2} \mathrm{~cm}^{-3}$ are used. We derived a molecular gas mass of $1.3 \times 10^{7} M_{\odot}$, and an area filling factor of $\sim 2 \%$.

The two-component fitting refers to a region of $45^{\prime \prime}$ in radius, which corresponds to a radius of $450 \mathrm{pc}$. The total molecular gas mass is derived to be $8.9 \times 10^{7} M_{\odot}$ for the best-fit result, which contains a gas mass of $6.6 \times 10^{7} M_{\odot}$ for the LE component, and $2.3 \times 10^{7} M_{\odot}$ for the HE component.

The molecular gas mass in Circinus has been debated for a long time. Using the $1.3 \mathrm{~mm}$ continuum, Siebenmorgen et al. (1997) derived a molecular gas mass of $1.6 \times 10^{8} M_{\odot}$ within their $3^{\prime} \times 4^{\prime}$ maps of the central region of Circinus. From the Galactic disk standard conversion factor $\left(2 \times 10^{20}\left(\mathrm{~K} \mathrm{~km} \mathrm{~s}^{-1}\right)^{-1}\right.$; Strong et al. 1988; Bolatto et al. 2013), the molecular gas mass derived from ${ }^{12} \mathrm{CO} J=1 \rightarrow 0$ reaches $1.6 \times 10^{9} M_{\odot}$ in the central 560 pc (e.g., Elmouttie et al. 1997; Curran et al. 1998). This would indicate that the molecular gas mass constitutes half of the dynamical mass in the central 560 pc region, which is more than the molecular gas mass fractions in most luminous galaxies and nuclear regions of normal S-F galaxies (e.g., Young \& Scoville 1991; Sakamoto et al. 1999). Hitschfeld et al. (2008) performed both Local Thermal Equilibrium (LTE) and LVG analysis with the lowest four transitions of $\mathrm{CO}$ and the $\mathrm{CI}$ transition. They found that the column density of $\mathrm{CO}$ is about $4-7 \times 10^{17} \mathrm{~cm}^{-2}$ in the central $560 \mathrm{pc}$ region, and this is $\sim 1 / 10$ of the column density $\left(N_{\mathrm{CO}}=3 \times 10^{18} \mathrm{~cm}^{-2}\right)$ derived from the standard conversion factor. This evidence implies that the standard conversion factor in Circinus is ten times lower than the Galactic disk value (e.g., Dahmen et al. 1998; Bell et al. 2007; Israel 2009a,b; Bolatto et al. 2013)

The best fitting in our two-component LVG modeling gives a total molecular gas mass of $\sim 9 \times 10^{7} M_{\odot}$ in the central $45^{\prime \prime}$ region, which corresponds to a standard conversion factor of $N\left(\mathrm{H}_{2}\right) / I_{\mathrm{COJ}=1 \rightarrow 0}=0.37 \times 10^{20} \mathrm{~cm}^{-2}\left(\mathrm{~K} \mathrm{~km} \mathrm{~s}^{-1}\right)^{-1}\left(\right.$ for $x_{\mathrm{CO}}=$ $8 \times 10^{-5}$ used here). The molecular gas mass determined from LVG modeling is about $60 \%$ of the mass of $1.6 \times 10^{8} M_{\odot}$ derived by the $1.3 \mathrm{~mm}$ continuum obtained in a larger region (Siebenmorgen et al. 1997). Mauersberger et al. (1996), Downes \& Solomon (1998), Papadopoulos \& Seaquist (1999), Israel (2009a), and Bolatto et al. (2013) also derived conversion factors significantly lower than the Galactic value by analyzing the low- $J$ CO emission in NGC 1068 and other galaxies with bright $\mathrm{CO}$ emission and high stellar surface density. This suggests that the lower conversion factor likely arises from gas being not virialized (e.g., Aalto et al. 1995; Dahmen et al. 1998; Narayanan et al. 2011).

\subsection{Molecular gas mass estimates using $C l$}

Atomic carbon (CI) could help circumvent the problem of defining a proper conversion factor because its emission traces molecular gas independently. The critical density of $\mathrm{CI}$ is $\approx 1 \times$ $10^{3} \mathrm{~cm}^{-3}$ (e.g., Tielens 2005), similar to that of ${ }^{12} \mathrm{CO} J=1 \rightarrow 0$, thus provides approximate thermalization at the densities reported here (see Tables 7, 9, and 10). Strong evidence shows that $\mathrm{C}$ I and $\mathrm{CO}$ luminosities have a tight correlation in galaxies, independent of physical environment, IR luminosity, or redshift (e.g., Papadopoulos \& Greve 2004; Zhang et al. 2007; Walter et al. 2011). This suggests that $C$ I emission arises from the same volume and shares similar excitation temperature as $\mathrm{CO}$ (e.g., Ikeda et al. 2002). Constant ratios between the column densities of $\mathrm{CI},{ }^{12} \mathrm{CO}$, and $\mathrm{H}_{2}$ are expected over a large range of physical conditions (e.g., Papadopoulos \& Greve 2004; Walter et al. 2011).

Constraining $\mathrm{H}_{2}$ column density with the optically thin $\mathrm{C} \mathrm{I}$ lines is an independent and robust way to probe the molecular 


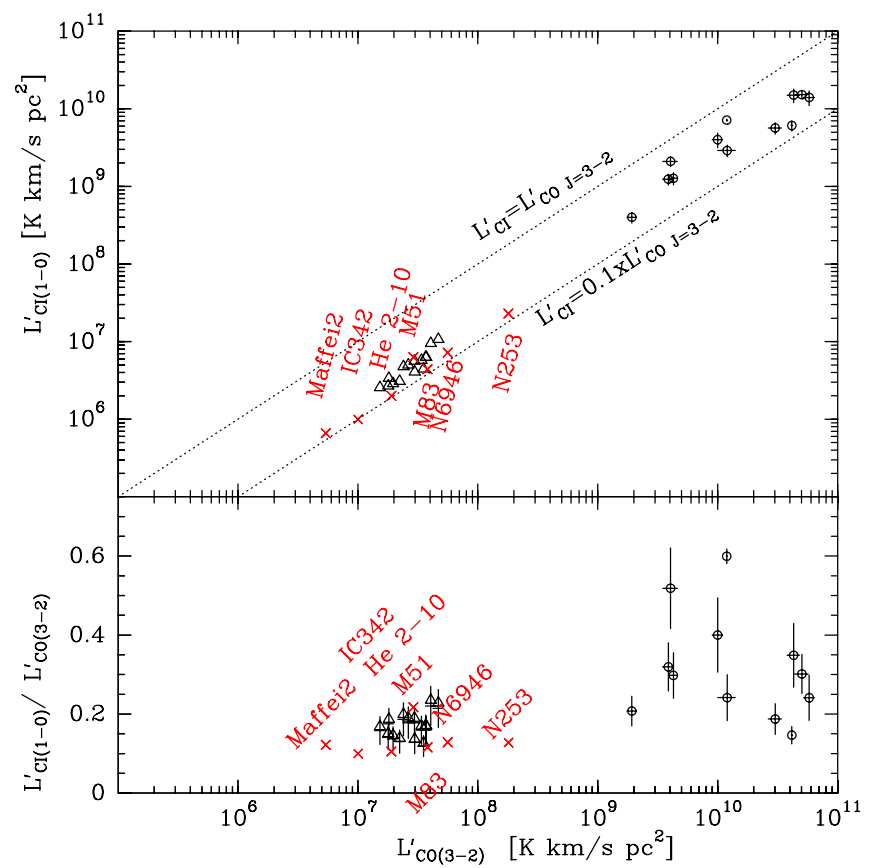

Fig. 10. $L_{\mathrm{CI}}^{\prime}$ as a function of $L_{\mathrm{CO} J=3 \rightarrow 2}^{\prime}$. The empty triangles show observed positions in Circinus. The empty circles show the galaxies at high redshift (Walter et al. 2011). The crosses represent results of nearby galaxies found in literature. NGC 6946 and M 83: (Israel \& Baas 2001), M 51: (Israel et al. 2006), Hennize 2-10 and NGC 253: (Bayet et al. 2004), IC 342: (Israel \& Baas 2003).

gas mass in galaxies. We can calculate the mass of $\mathrm{C}$ I following Weiß et al. (2005):

$M(\mathrm{CI})=1.9 \times 10^{-4} Q\left(T_{\mathrm{ex}}\right) \mathrm{e}^{23.6 / T_{\text {ex }}} L^{\prime}\left(\mathrm{CI}^{3} P_{1} \rightarrow^{3} P_{0}\right)\left[M_{\odot}\right]$,

where $Q\left(T_{\mathrm{ex}}\right)=1+3 \mathrm{e}^{-T_{1} / T_{\mathrm{ex}}}+5 \mathrm{e}^{-T_{2} / T_{\mathrm{ex}}}$ is the C I partition function, and $T_{1}=23.6 \mathrm{~K}$ and $T_{2}=62.5 \mathrm{~K}$ are the energies above the ground state. The $[\mathrm{CI}] /[\mathrm{H} 2]$ abundance chosen here is $x_{\mathrm{C}_{\mathrm{I}}}=$ $5 \times 10^{-5}$ (Weiß et al. 2005). We adopt an excitation temperature of $30 \mathrm{~K}$ derived from the LE component in the two-component LVG fittings (Table 9). Assuming $T_{\mathrm{ex}}=T_{\text {kin }}=30 \mathrm{~K}$, we derive a molecular gas mass of $8.3 \times 10^{7} M_{\odot}$. If $T_{\text {ex }}=60 \mathrm{~K}$ from the HE component is adopted, the molecular gas mass is $8.9 \times$ $10^{7} M_{\odot}$. These masses are, within the errors, consistent with the result derived from our LVG solution, $9 \times 10^{7} M_{\odot}$ (Sect. 4.7).

\subsection{Luminosities of $C$ I and ${ }^{3} P_{1} \rightarrow{ }^{3} P_{0}$}

In Fig. 2, we present the spectra of $\mathrm{CI}$ and ${ }^{12} \mathrm{CO} J=3 \rightarrow 2$ observed in the central region of Circinus. The line profiles of the two species are similar. In the following, we calculate the line luminosities $\left(L_{\text {line }}^{\prime}\right)$ of $\mathrm{CO}$ and $\mathrm{C} \mathrm{I}$, and compare them with nearby galaxies and high- $z$ systems. We determine the line luminosity following the definition in Solomon et al. (1992):

$$
\frac{L_{\text {line }}^{\prime}}{\left(\mathrm{K} \mathrm{km} \mathrm{s}^{-1} \mathrm{pc}^{2}\right)}=3.25 \times 10^{7} S_{\text {line }} \Delta v v_{\text {obs }}^{-2} D_{\mathrm{L}}^{2}(1+z)^{-3} \text {, }
$$

where $L^{\prime}$ is the line luminosity in $\mathrm{K} \mathrm{km} \mathrm{s}^{-1} \mathrm{pc}^{2}, S_{\text {line }} \Delta v$ is the velocity integrated flux density in Jy $\mathrm{km} \mathrm{s}^{-1}, D_{\mathrm{L}}$ denotes the luminosity distance in $\mathrm{Mpc}$, and $v_{\mathrm{obs}}$ represents the observing frequency in GHz. The $L_{\text {line }}^{\prime}$ ratios stand for ratios of the intrinsic brightness temperatures.

In Fig. 10, we plot the $\mathrm{C}$ I line luminosity as a function of ${ }^{12} \mathrm{CO} J=3 \rightarrow 2$ luminosity. We combined the $\mathrm{C}$ I data from the literature, including the $\mathrm{CI}$ detections in nearby and highredshift galaxies (e.g., Walter et al. 2011). The luminosities of $\mathrm{CI}$ and ${ }^{12} \mathrm{CO} J=3 \rightarrow 2$ match each other and the scatter of the ratios lies within an order of magnitude (the dashed diagonal lines). The correlation derived from multiple position in Circinus basically follows the same trend found in high-redshift galaxies.

We find that the $\mathrm{CI}$ to ${ }^{12} \mathrm{CO} J=3 \rightarrow 2$ luminosity ratios $R_{\mathrm{CI} / \mathrm{CO} 32}$ in nearby galaxies (red crosses) are lower than those found in the high-redshift galaxies and the AGN hosting galaxies (i.e., Circinus and $M$ 51). An average ratio of $R_{\mathrm{CI} / \mathrm{CO} 32}=0.17 \pm 0.03$ is found in Circinus, and this is only about half of the average ratio found at highredshift (Walter et al. $2011,0.32 \pm 0.13)$. In the quiescent nearby galaxies, $R_{\mathrm{CI} / \mathrm{CO} 32}$ are mostly close to 0.1 . In the central positions of M 51 and Circinus, the $R_{\mathrm{CI} / \mathrm{CO} 32}$ ratios are both $\sim 0.2$, about two times higher than those in quiescent galaxies. These high $R_{\mathrm{CI} / \mathrm{CO} 32}$ ratios are likely caused by the enhanced gas excitation due to the AGN activities.

\section{Summary and conclusions}

We present new APEX mapping observations of ${ }^{12} \mathrm{CO} J=3 \rightarrow$ $2,4 \rightarrow 3,6 \rightarrow 5,7 \rightarrow 6$ and C I $1 \rightarrow 0$ in the central region of the Circinus galaxy. These data are to date the highest transitions published. All these lines reveal extended strong emission and similar kinematic structures. We find strong ${ }^{12} \mathrm{CO} J=6 \rightarrow 5$ and $7 \rightarrow 6$ emission not only in the nuclear region, but in the gas-rich, star-forming (S-F) ring region at galactocentric diameter of $18^{\prime \prime}<D<45^{\prime \prime}$ as well. The latter region contributes about $35-45 \%$ of the measured high- $J$ CO emission. With the CO maps we are able to decompose the gas excitation spatially.

By using radiation transfer analysis we find two distinct areas with different gas excitation conditions: the $18^{\prime \prime}$ nuclear region and the $\mathrm{S}-\mathrm{F}$ ring within $18^{\prime \prime}<R<45^{\prime \prime}$. Our main results are as follows:

1) With a single excitation component, we use APEX ${ }^{12} \mathrm{CO}$ and ${ }^{13} \mathrm{CO}$ detections $(J \geq 3)$ to perform a LVG modeling. We derive $n_{\mathrm{H}_{2}} \sim 10^{3.2} \mathrm{~cm}^{-3}, T_{\text {kin }} \sim 200 \mathrm{~K}, \mathrm{~d} v / \mathrm{d} r \sim 3.0 \mathrm{~km} \mathrm{~s}^{-1} \mathrm{pc}^{-1}$, and $M_{\mathrm{H}_{2}} \sim 1.3 \times 10^{7} M_{\odot}$ in the central $18^{\prime \prime}$ region, which accounts for $\sim 15 \%$ of the total molecular gas mass in the central gas-rich $45^{\prime \prime}$ region in Circinus.

2) Combined with low- $J \mathrm{CO}$ data in the literature, we perform two-component LVG modeling in the central $45^{\prime \prime}$ diameter region, and in the S-F ring. We find two excitation components that can fit the measurements in the whole region, one with $n_{\mathrm{H}_{2}} \sim 10^{3.0} \mathrm{~cm}^{-3}, T_{\text {kin }} \sim 30 \mathrm{~K}, \mathrm{~d} v / \mathrm{d} r \sim 6 \mathrm{~km} \mathrm{~s}^{-1} \mathrm{pc}^{-1}$, and $M_{\mathrm{H}_{2}} \sim 6.6 \times 10^{7} M_{\odot}$, and the other with $n_{\mathrm{H}_{2}} \sim 10^{4.2} \mathrm{~cm}^{-3}$, $T_{\text {kin }} \sim 60 \mathrm{~K}, \mathrm{~d} v / \mathrm{d} r \sim 50 \mathrm{~km} \mathrm{~s}^{-1} \mathrm{pc}^{-1}$, and $M_{\mathrm{H}_{2}} \sim 2.3 \times 10^{7} M_{\odot}$. In the ring region, the high density component represents a smaller fraction $(\sim 13 \%)$ of the total gas mass. All these gas components are supervirialized.

3) We find the molecular gas mass of Circinus is $\sim 0.9 \times 10^{8} M_{\odot}$ in the $45^{\prime \prime}$ region. This is consistent with the gas mass derived from C I $\left(\sim 0.9 \times 10^{8} M_{\odot}\right)$ and is $\sim 60 \%$ of the gas mass obtained using submm continuum in a larger area $\left(1.6 \times 10^{8} M_{\odot}\right)$. A gas mass of about $\sim 1.3 \times 10^{7} M_{\odot}$ is found in the central $18^{\prime \prime}$ nuclear region, and $\sim 7.5 \times 10^{7} M_{\odot}$ is located in the surrounding ring. In the $45^{\prime \prime}$ region, we thus derive a conversion factor of $N\left(\mathrm{H}_{2}\right) / I_{\mathrm{COJ}=1 \rightarrow 0}=0.37 \times$ $10^{20} \mathrm{~cm}^{-2}\left(\mathrm{~K} \mathrm{~km} \mathrm{~s}^{-1}\right)^{-1}$, which is about $1 / 5$ of the Galactic disk value.

4) We find the average luminosity ratio between $\mathrm{C}$ I $(1 \rightarrow 0)$ and ${ }^{12} \mathrm{CO} J=3 \rightarrow 2\left(R_{\mathrm{CI} / \mathrm{CO} 32}\right)$ in Circinus to be 0.2 , about twice 
the average value found in nearby normal galaxies (Gerin \& Phillips 2000, 0.1). This is near the low end of what is observed in high-redshift systems (Walter et al. 2011, 0.29).

Acknowledgements. We thank the anonymous referee for his/her very thorough reading of the draft, and the very detailed comments that have significantly improved the quality of the paper. We are grateful to the staff at the APEX Station of MPIfR for their assistance during the observations. Z.Z. thanks J. Z. Wang, L. J. Shao and K. J. Li for their constructive discussions. Z.Z. acknowledges support from the European Research Council (ERC) in the form of Advanced Grant, COSMICISM. This work was partly supported by NSF China grants \#11173059 and \#11390373, and CAS No. XDB09000000. Y.A. acknowledges support from the grant 11003044/11373007 from the National Natural Science Foundation of China.

\section{References}

Aalto, S., Johansson, L. E. B., Booth, R. S., \& Black, J. H. 1991, A\&A, 249, 323 Aalto, S., Booth, R. S., Black, J. H., \& Johansson, L. E. B. 1995, A\&A, 300, 369 Andrae, R., Schulze-Hartung, T., \& Melchior, P. 2010, unpublished [arXiv: 1012.3754]

Bayet, E., Gerin, M., Phillips, T. G., \& Contursi, A. 2004, A\&A, 427, 45 Bayet, E., Gerin, M., Phillips, T. G., \& Contursi, A. 2009, MNRAS, 399, 264 Bell, T. A., Viti, S., \& Williams, D. A. 2007, MNRAS, 378, 983 Bertoldi, F., \& McKee, C. F. 1992, ApJ, 395, 140

Blain, A. W., Frayer, D. T., Bock, J. J., \& Scoville, N. Z. 2000, MNRAS, 313, 559

Bland-Hawthorn, J., Gallimore, J. F., Tacconi, L. J., et al. 1997, Ap\&SS, 248, 9 Bolatto, A. D., Wolfire, M., \& Leroy, A. K. 2013, ARA\&A, 51, 207 Bonnell, I. A., \& Rice, W. K. M. 2008, Science, 321, 1060 Bradford, C. M., Stacey, G. J., Nikola, T., et al. 2005, ApJ, 623, 866 Braine, J., Combes, F., Casoli, F., et al. 1993, A\&AS, 97, 887

Bryant, P. M., \& Scoville, N. Z. 1996, ApJ, 457, 678

Bundy, K., Georgakakis, A., Nandra, K., et al. 2008, ApJ, 681, 931

Carilli, C. L., \& Walter, F. 2013, ARA\&A, 51, 105

Carilli, C. L., Daddi, E., Riechers, D., et al. 2010, ApJ, 714, 1407

Combes, F., Maoli, R., \& Omont, A. 1999, A\&A, 345, 369

Curran, S. J., Johansson, L. E. B., Rydbeck, G., \& Booth, R. S. 1998, A\&A, 338, 863

Curran, S. J., Rydbeck, G., Johansson, L. E. B., \& Booth, R. S. 1999, A\&A, 344, 767

Curran, S. J., Johansson, L. E. B., Bergman, P., Heikkilä, A., \& Aalto, S. 2001, A\&A, 367, 457

Curran, S. J., Koribalski, B. S., \& Bains, I. 2008, MNRAS, 389, 63

Dahmen, G., Huttemeister, S., Wilson, T. L., \& Mauersberger, R. 1998, A\&A, 331, 959

Downes, D., \& Solomon, P. M. 1998, ApJ, 507, 615

Dumke, M., Nieten, C., Thuma, G., Wielebinski, R., \& Walsh, W. 2001, A\&A, 373,853

Elmouttie, M., Haynes, R. F., \& Jones, K. L. 1997, PASA, 14, 140

Flower, D. R. 2001, MNRAS, 328, 147

For, B.-Q., Koribalski, B. S., \& Jarrett, T. H. 2012, MNRAS, 425, 1934

Freeman, K. C., Karlsson, B., Lynga, G., et al. 1977, A\&A, 55, 445

Frerking, M. A., Langer, W. D., \& Wilson, R. W. 1982, ApJ, 262, 590

Gallimore, J. F., Henkel, C., Baum, S. A., et al. 2001, ApJ, 556, 694

Geach, J. E., \& Papadopoulos, P. P. 2012, ApJ, 757, 156

Gerin, M., \& Phillips, T. G. 2000, ApJ, 537, 644

Goldreich, P., \& Kwan, J. 1974, ApJ, 189, 441

Greenhill, L. J., Kondratko, P. T., Lovell, J. E. J., et al. 2003, ApJ, 582, L11

Güsten, R., Philipp, S. D., Weiß, A., \& Klein, B. 2006, A\&A, 454, L115

Hagiwara, Y., Miyoshi, M., Doi, A., \& Horiuchi, S. 2013, ApJ, 768, L38

Hailey-Dunsheath, S., Nikola, T., Stacey, G. J., et al. 2008, ApJ, 689, L109

Hailey-Dunsheath, S., Sturm, E., Fischer, J., et al. 2012, ApJ, 755, 57

Harada, N., Thompson, T. A., \& Herbst, E. 2013, ApJ, 765, 108

Helfer, T. T., Thornley, M. D., Regan, M. W., et al. 2003, ApJS, 145, 259

Henkel, C., \& Mauersberger, R. 1993, A\&A, 274, 730

Henkel, C., Asiri, H., Ao, Y., et al. 2014, A\&A, 565, A3

Hitschfeld, M., Aravena, M., Kramer, C., et al. 2008, A\&A, 479, 75

Ikeda, M., Oka, T., Tatematsu, K., Sekimoto, Y., \& Yamamoto, S. 2002, ApJS, 139,467

Israel, F. P. 1992, A\&A, 265, 487

Israel, F. P. 2009a, A\&A, 493, 525

Israel, F. P. 2009b, A\&A, 506, 689
Israel, F. P., \& Baas, F. 2001, A\&A, 371, 433

Israel, F. P., \& Baas, F. 2003, A\&A, 404, 495

Israel, F. P., Tilanus, R. P. J., \& Baas, F. 2006, A\&A, 445, 907

Johansson, L. E. B., Aalto, S., Booth, R. S., \& Rydbeck, G. 1991, in Dynamics of Disc Galaxies, ed. B. Sundelius, 249

Jones, K. L., Koribalski, B. S., Elmouttie, M., \& Haynes, R. F. 1999, MNRAS, 302,649

Kamenetzky, J., Glenn, J., Maloney, P. R., et al. 2011, ApJ, 731, 83

Kamenetzky, J., Glenn, J., Rangwala, N., et al. 2012, ApJ, 753, 70

Kasemann, C., Güsten, R., Heyminck, S., et al. 2006, in SPIE Conf. Ser., 6275

Kennicutt, R. C., \& Evans, N. J. 2012, ARA\&A, 50, 531

Klein, B., Philipp, S. D., Krämer, I., et al. 2006, A\&A, 454, L29

Krips, M., Martín, S., Eckart, A., et al. 2011, ApJ, 736, 37

Krumholz, M. R., \& McKee, C. F. 2005, ApJ, 630, 250

Langer, W. D., \& Penzias, A. A. 1993, ApJ, 408, 539

Lu, N., Zhao, Y., Xu, C. K., et al. 2014, ApJ, 787, L23

Maiolino, R., Krabbe, A., Thatte, N., \& Genzel, R. 1998, ApJ, 493, 650

Mao, R. Q., Henkel, C., Schulz, A., et al. 2000, A\&A, 358, 433

Marconi, A., Moorwood, A. F. M., Origlia, L., \& Oliva, E. 1994, The Messenger, 78,20

Mauersberger, R., Henkel, C., Wielebinski, R., Wiklind, T., \& Reuter, H.-P. 1996, A\&A, 305, 421

Narayanan, D., Krumholz, M., Ostriker, E. C., \& Hernquist, L. 2011, MNRAS, 418, 664

Omont, A., Petitjean, P., Guilloteau, S., et al. 1996, Nature, 382, 428

Ott, J., Weiß, A., Staveley-Smith, L., Henkel, C., \& Meier, D. S. 2014, ApJ, 785, 55

Papadopoulos, P. P., \& Greve, T. R. 2004, ApJ, 615, L29

Papadopoulos, P. P., \& Seaquist, E. R. 1999, ApJ, 516, 114

Papadopoulos, P. P., van der Werf, P., Xilouris, E., Isaak, K. G., \& Gao, Y. 2012a, ApJ, 751, 10

Papadopoulos, P. P., van der Werf, P. P., Xilouris, E. M., et al. 2012b, MNRAS, 426, 2601

Pérez-Beaupuits, J. P., Wada, K., \& Spaans, M. 2011, ApJ, 730, 48

Rangwala, N., Maloney, P. R., Glenn, J., et al. 2011, ApJ, 743, 94

Rigopoulou, D., Hurley, P. D., Swinyard, B. M., et al. 2013, MNRAS, 434, 2051

Sakamoto, K., Okumura, S. K., Ishizuki, S., \& Scoville, N. Z. 1999, ApJ, 525, 691

Sani, E., Lutz, D., Risaliti, G., et al. 2010, MNRAS, 403, 1246

Schinnerer, E., Eckart, A., Tacconi, L. J., Genzel, R., \& Downes, D. 2000, ApJ, 533,850

Schlegel, D. J., Finkbeiner, D. P., \& Davis, M. 1998, ApJ, 500, 525

Schöier, F. L., van der Tak, F. F. S., van Dishoeck, E. F., \& Black, J. H. 2005, A\&A, 432, 369

Scoville, N. Z., \& Solomon, P. M. 1974, ApJ, 187, L67

Siebenmorgen, R., Moorwood, A., Freudling, W., \& Kaeufl, H. U. 1997, A\&A, 325,450

Solomon, P. M., Radford, S. J. E., \& Downes, D. 1992, Nature, 356, 318

Spinoglio, L., Pereira-Santaella, M., Busquet, G., et al. 2012, ApJ, 758, 108

Strong, A. W., Bloemen, J. B. G. M., Dame, T. M., et al. 1988, A\&A, 207, 1

Tacconi, L. J., Gallimore, J. F., Genzel, R., Schinnerer, E., \& Downes, D. 1997, Ap\&SS, 248, 59

Tan, Q.-H., Gao, Y., Zhang, Z.-Y., \& Xia, X.-Y. 2011, RA\&A, 11, 787

Tielens, A. G. G. M. 2005, The Physics and Chemistry of the Interstellar Medium (Cambridge University Press)

Tristram, K. R. W., Meisenheimer, K., Jaffe, W., et al. 2007, A\&A, 474, 837

Tsai, M., Hwang, C.-Y., Matsushita, S., Baker, A. J., \& Espada, D. 2012, ApJ, 746,129

Tully, R. B., Rizzi, L., Shaya, E. J., et al. 2009, AJ, 138, 323

van der Tak, F. F. S., Black, J. H., Schöier, F. L., Jansen, D. J., \& van Dishoeck, E. F. 2007, A\&A, 468, 627

van der Werf, P. P., Isaak, K. G., Meijerink, R., et al. 2010, A\&A, 518, L42

Walter, F., Weiß, A., Downes, D., Decarli, R., \& Henkel, C. 2011, ApJ, 730, 18

Wang, R., Carilli, C. L., Neri, R., et al. 2010, ApJ, 714, 699

Ward, J. S., Zmuidzinas, J., Harris, A. I., \& Isaak, K. G. 2003, ApJ, 587, 171

Weiß, A., Downes, D., Henkel, C., \& Walter, F. 2005, A\&A, 429, L25

White, R. E. 1977, ApJ, 211, 744

Wilson, A. S., Shopbell, P. L., Simpson, C., et al. 2000, AJ, 120, 1325

Wilson, C. D., Warren, B. E., Irwin, J., et al. 2011, MNRAS, 410, 1409

Wilson, T. L., \& Matteucci, F. 1992, A\&ARv, 4, 1

Wilson, T. L., \& Rood, R. 1994, ARA\&A, 32, 191

Yang, B., Stancil, P. C., Balakrishnan, N., \& Forrey, R. C. 2010, ApJ, 718, 1062

Young, J. S., \& Scoville, N. Z. 1991, ARA\&A, 29, 581

Zhang, J. S., Henkel, C., Mauersberger, R., et al. 2007, A\&A, 465, 887

Pages 15 to 16 are available in the electronic edition of the journal at http://www . aanda.org 


\section{Appendix A: Virialized gas state}

The gravitational potential of the densely packed stars and the nearby supermassive nuclear engine in the central region of a massive galaxy may cause significant velocity gradients along lines of sight, which can be well in excess of what would be found in a normal cloud near virial equilibrium. Therefore the velocity gradient expected in the virialized gas motion can be taken as a lower limit. The ratio between the measured velocity gradient and that calculated from virial equilibrium is defined by

$K_{\mathrm{vir}}=\frac{(\mathrm{d} v / \mathrm{d} r)_{\mathrm{LVG}}}{(\mathrm{d} v / \mathrm{d} r)_{\mathrm{vir}}}$

The virialized velocity gradient is given by

$$
\begin{aligned}
\left(\frac{\mathrm{d} V}{\mathrm{~d} r}\right)_{\mathrm{VIR}} & \approx \frac{\delta v_{\mathrm{vir}}}{2 R}=\left(\alpha \frac{\pi G \mu}{3}\right)^{1 / 2}\langle n\rangle^{1 / 2} \\
& \sim 0.65 \alpha^{0.5}\left(\frac{\langle n\rangle}{10^{3} \mathrm{~cm}^{-3}}\right)^{1 / 2} \mathrm{~km} \mathrm{~s}^{-1} \mathrm{pc}^{-1},
\end{aligned}
$$

where $\mu$ is the mean particle mass, $G$ is the gravitational constant, $\langle n\rangle$ is the mean number density of the cloud, and $\alpha$ is a constant between 0.5 to 3 depending on the assumed density profile (Bryant \& Scoville 1996). For a cloud with assumed density of $10^{5} \mathrm{~cm}^{-3}$, and with the largest value of $\alpha=3$, the estimated $(\mathrm{d} v / \mathrm{d} r)$ is about $10 \mathrm{~km} \mathrm{~s}^{-1} \mathrm{pc}^{-1}$. For more diffuse gas with a density of $10^{3} \mathrm{~cm}^{-3}$ and $\alpha=0.5,(\mathrm{~d} v / \mathrm{d} r)$ is around $0.5 \mathrm{~km} \mathrm{~s}^{-1} \mathrm{pc}^{-1}$. Molecular gas close to the central massive black hole will be strongly influenced by gravity (e.g., Bonnell \& Rice 2008), thus could be subvirialized $\left(K_{\mathrm{vir}}<1\right)$. However such an effect is likely obvious only within a few tenths pc in the center. On the other hand, the tidal shear produced by the black hole would also increase the instability of molecular gas, where $K_{\mathrm{vir}}>1$.

\section{Appendix B: Likelihood analysis of the single-component fitting}

In the following, we analyze possible solution ranges for the central $18^{\prime \prime}$ of the Circinus galaxy and corresponding physical conditions satisfying maximum likelihood achievable in the set of all combinations parameters (see also Sect. 4.2.1). We caution, however, that these findings - in particular the numbers shown below - are rather uncertain, and will be only indicative.

Instead of the Bayesian probability, which is the integral of all probabilities in the parameter space (e.g., Weiß et al. 2005; Hailey-Dunsheath et al. 2012; Kamenetzky et al. 2011; Rangwala et al. 2011), we analyze the trend of the solutions with the highest likelihood. The maximum likelihood function of a given parameter (or given parameters) is based on the best fitting results in the whole parameter space.

Figure B.1 (upper panel) shows the maximum likelihood as a function of velocity gradient in a range of $1 \mathrm{~km} \mathrm{~s}^{-1} \mathrm{pc}^{-1} \leq \mathrm{d} v / \mathrm{d} r \leq 10^{3} \mathrm{~km} \mathrm{~s}^{-1} \mathrm{pc}^{-1}$. We plot the corresponding values of $n_{\mathrm{H}_{2}}$ and $T_{\mathrm{kin}}$ of the best fit for each given velocity gradient (lower panel). Over the modeled $\mathrm{d} v / \mathrm{d} r$ range, $T_{\text {kin }}$ and $n_{\mathrm{H}_{2}}$ vary by about an order of magnitude. The likelihood drops below half of the peak value when $\mathrm{d} v / \mathrm{d} r$ is beyond $10^{1.8} \mathrm{~km} \mathrm{~s}^{-1} \mathrm{pc}^{-1}$, where the solutions have relative high temperature and low density. This suggests that reasonable models are not likely to have a $\mathrm{d} v / \mathrm{d} r$ higher than $60 \mathrm{~km} \mathrm{~s}^{-1} \mathrm{pc}^{-1}$ because then even the best fitting result shows poor fits to the measurements.

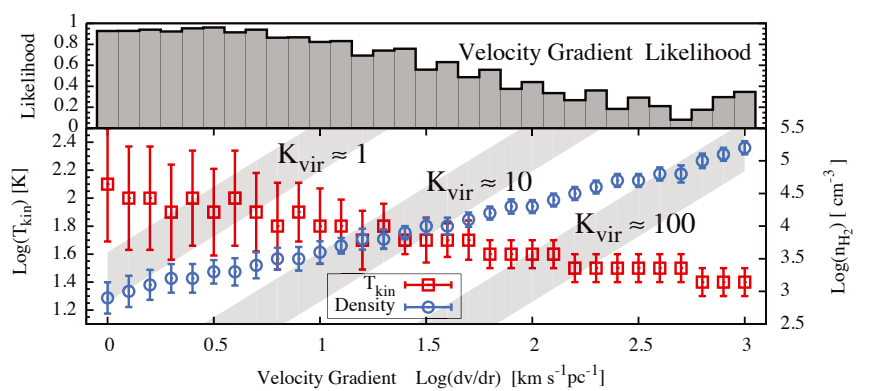

Fig. B.1. Upper panel: maximum likelihood as a function of the velocity gradient $\mathrm{d} v / \mathrm{d} r$ for single-component LVG fitting. Lower panel: bestfit values of density (blue circles) and temperature (red squares) for a given velocity gradient as functions of velocity gradient, with error bars showing a $1 \sigma$ range of the likelihood distribution. The gray regions have $K_{\text {vir }} \sim 1,10$, and 100 .

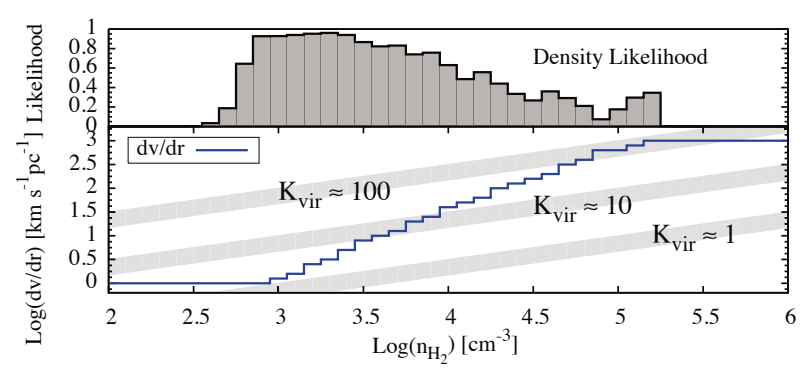

Fig. B.2. Upper panel: maximum likelihood as a function density for single-component LVG fitting. Lower panel: best-fit velocity gradient for a given density as a function of density. The gray regions have $K_{\mathrm{vir}} \sim$ 1,10 , and 100 .

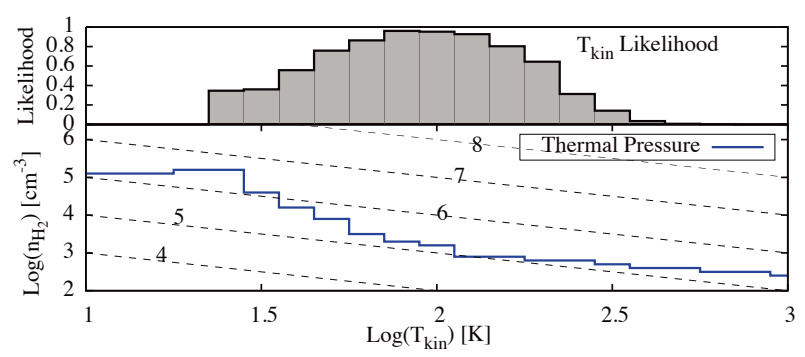

Fig. B.3. Upper panel: maximum likelihood as a function of temperature for single-component LVG fitting. Lower panel: best-fit density for a given temperature as a function of temperature. The dashed lines show thermal pressure, $\log \left(n_{\mathrm{H}_{2}} \times T_{\text {kin }}\right)$, in units of $\mathrm{K} \mathrm{cm}^{-3}$.

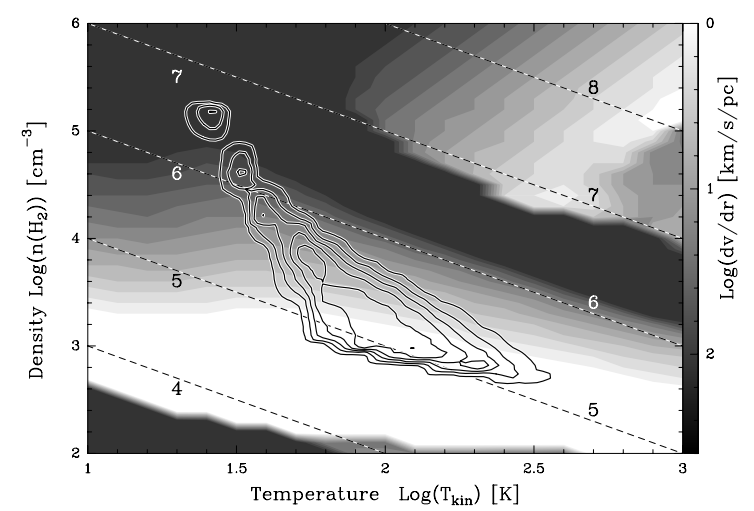

Fig. B.4. The contours show the distributions of the maximum likelihood for a given density and temperature in the single-component LVG fitting. Contours are drawn from 0.1 to 0.9 by 0.2 . Background gray scale levels show the velocity gradient associated with the best LVG fitting results, for each given temperature and density. The dashed lines indicate the thermal pressure $\log \left(n_{\mathrm{H}_{2}} \times T_{\text {kin }}\right)$ in units of $\mathrm{K} \mathrm{cm}^{-3}$. 


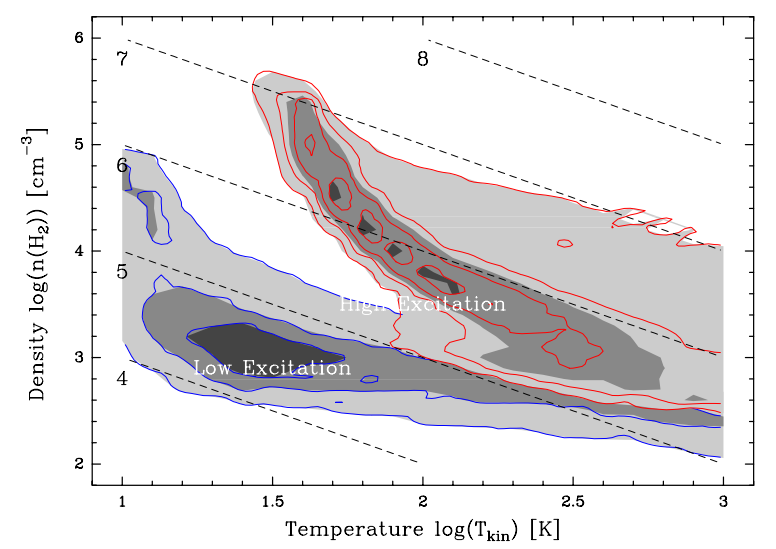

Fig. C.1. Background gray scale images show the maximum likelihood distributions of temperature and density, derived from the twocomponent LVG modelings. The gray levels are from 0.1 to 0.9 with a spacing of 0.2. The low- and high-excitation components are plotted in blue and red contours. The dashed lines indicate thermal pressure, $\log \left(n_{\mathrm{H}_{2}} \times T_{\text {kin }}\right)$, in units of $\mathrm{K} \mathrm{cm}^{-3}$.

Figure B.2 shows the maximum likelihood as a function of $n_{\mathrm{H}_{2}}$ in a $\mathrm{H}_{2}$ density range from $10^{2} \mathrm{~cm}^{-2}$ to $10^{6} \mathrm{~cm}^{-2}$. We plot the corresponding $\mathrm{d} v / \mathrm{d} r$ of the best fits as a function of density. The solutions are found over a broad range of velocity gradients, which increase almost linearly as density increases. Solutions with high densities also have high velocity gradients. But the density is not likely to be higher than $10^{4.5} \mathrm{~cm}^{-3}$ where the likelihood is dropping below half of the peak value and $K_{\mathrm{vir}}$ exceeds ten. This implies that models with high density solutions are highly supervirialized and are not bound by self-gravity.

Figure B.3 shows the maximum likelihood as a function of $T_{\text {kin }}$ from $10 \mathrm{~K}$ to $10^{3} \mathrm{~K}$. The thermal pressure $P=n_{\mathrm{H}_{2}} \times T_{\text {kin }}$ of the best fitting results is presented for given temperatures. The thermal pressure decreases by an order of magnitude when $T_{\text {kin }}$ increases from a few tens of $\mathrm{K}$ to about $200 \mathrm{~K}$. This indicates that the solutions of high temperature will have low thermal pressure because of the corresponding low density of these solutions.

In Fig. B.4, we show as contours the density-temperature likelihood distribution of the LVG modeling. The gray scale background displays the velocity gradient associated with the best fitting results, at given temperatures and densities. The contours present a banana-shaped likelihood distribution, which is mainly caused by the degeneracy between temperature and density. In the contour map, thermal pressure almost stays constant along the ridge of the distribution. Both density and temperature vary by two orders of magnitude within the $50 \%$ contour. The likelihood distribution covers a range of thermal pressure from $10^{4.8}$ to $10^{6.5} \mathrm{~K} \mathrm{~cm}^{-3}$ and peaks at $\sim 10^{5.2} \mathrm{~K} \mathrm{~cm}^{-3}$. From the map of the associated velocity gradients in the background, $\mathrm{d} v / \mathrm{d} r$ increases with $n_{\mathrm{H}_{2}}$, and decreases with $T_{\text {kin }}$. Most good solutions have small $\mathrm{d} v / \mathrm{d} r$ between $1 \mathrm{~km} \mathrm{~s}^{-1} \mathrm{pc}^{-1}$ and $10 \mathrm{~km} \mathrm{~s}^{-1} \mathrm{pc}^{-1}$.

\section{Appendix C: Likelihood analysis of the two-component fitting}

In Fig. C.1, we show the maximum likelihood distribution of both components in our modeling, with contours of the enclosed probability. Both distributions have banana shapes that are mainly caused by the degeneracy between $T_{\text {kin }}$ and $n_{\mathrm{H}_{2}}$. We find that both distributions are characterized by component specific thermal pressures. The thermal pressure of the HE component is about one order of magnitude higher than that of the LE component.

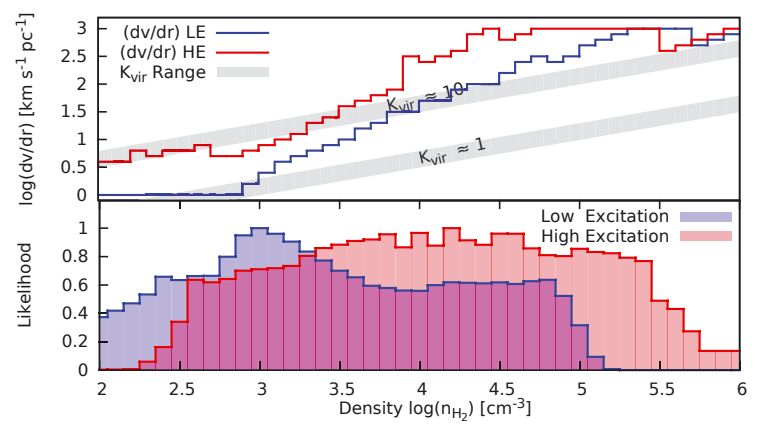

Fig. C.2. Upper panel: velocity gradients of the best fitting results as functions of density, derived from the two-component LVG modeling. The gray regions have $K_{\mathrm{vir}} \sim 1$ and 10. Lower panel: maximum likelihood as functions of the densities of both excitation components. The low- and high-excitation components are plotted in blue and red shadows, respectively.

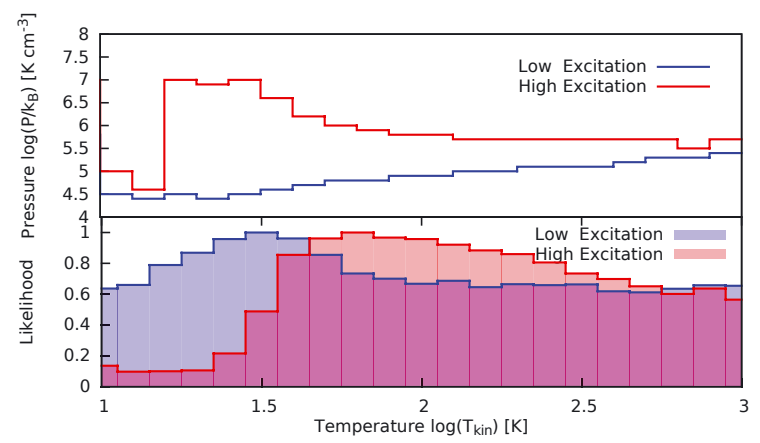

Fig. C.3. Upper panel: thermal pressure $\left(\log \left(n_{\mathrm{H}_{2}} \times T_{\text {kin }}\right)\right)$ of the best fitting results as functions of density, derived from the two-component LVG modeling. Lower panel: maximum likelihood as functions of temperatures for both excitation components. The low- and high-excitation components are plotted in blue and red shadows, respectively.

The HE component shows a steep slope in the high density and low temperature regime, and a flat slope at the high temperature side with a very broad range of $T_{\text {kin }}$ solutions. This indicates that the density is not tightly constrained for the HE component. The HE component has a best-fit $\mathrm{d} v / \mathrm{d} r$ of $\sim 50 \mathrm{~km} \mathrm{~s}^{-1} \mathrm{pc}^{-1}$, which is about 10 times higher than that of the LE component, where $\sim 6 \mathrm{~km} \mathrm{~s}^{-1} \mathrm{pc}^{-1}$ is the best fitting result. General fitting results of both excitation components are listed in Table 9.

Figure C.2 shows the density likelihood as functions of both excitation components (lower panel), and the corresponding velocity gradient of the best fittings for given densities (upper panel). The higher the density, the larger the velocity gradient for both components. Solutions with higher densities also have higher $K_{\mathrm{vir}}$. Molecular gas in such conditions has very violent motions and high temperature. Unless the HE component adopt a low density solution of $\sim 10^{3.5} \mathrm{~cm}^{-3}, K_{\mathrm{vir}}$ is always higher than unity. The density range of the HE component is much wider than that of the LE component, which is due to the high degeneracy between $n_{\mathrm{H}_{2}}, T_{\text {kin }}$, and $\mathrm{d} v / \mathrm{d} r$, and less constrained for the high- $J$ transitions. In Fig. C.3, the temperatures of both components are not well constrained although the likelihood curve of the LE component looks narrower and peaks at lower temperature $(\sim 40 \mathrm{~K})$ than that of the higher temperature $(\sim 50 \mathrm{~K})$. The thermal pressure drops when the temperature increases, and stays nearly constant when the temperatures of both components are higher than $100 \mathrm{~K}$. 